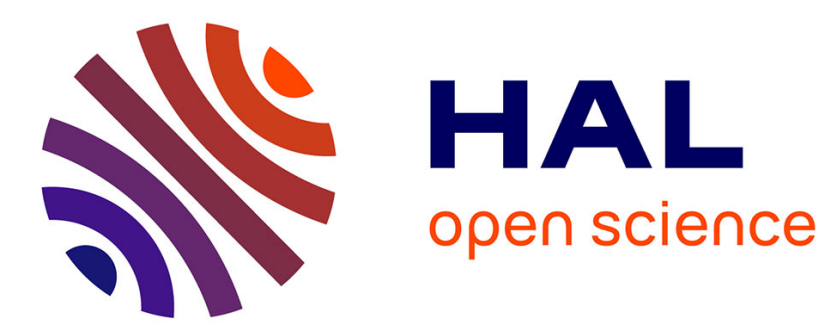

\title{
Precoded Optical Spatial Modulation for Indoor Visible Light Communications
}

Yongyang Li, Ping Yang, Marco Di Renzo, Yue Xiao, Ming Xiao, Wei Xiang

\section{To cite this version:}

Yongyang Li, Ping Yang, Marco Di Renzo, Yue Xiao, Ming Xiao, et al.. Precoded Optical Spatial Modulation for Indoor Visible Light Communications. IEEE Transactions on Communications, 2020, 69, pp.2518 - 2531. 10.1109/tcomm.2020.3041766 . hal-03377232

\section{HAL Id: hal-03377232 \\ https://hal.science/hal-03377232}

Submitted on 14 Oct 2021

HAL is a multi-disciplinary open access archive for the deposit and dissemination of scientific research documents, whether they are published or not. The documents may come from teaching and research institutions in France or abroad, or from public or private research centers.
L'archive ouverte pluridisciplinaire HAL, est destinée au dépôt et à la diffusion de documents scientifiques de niveau recherche, publiés ou non, émanant des établissements d'enseignement et de recherche français ou étrangers, des laboratoires publics ou privés. 


\title{
Precoded Optical Spatial Modulation for Indoor Visible Light Communications
}

\author{
Yongyang $\mathrm{Li}^{\circledR}$, Ping Yang ${ }^{\circledR}$, Senior Member, IEEE, Marco Di Renzo, Fellow, IEEE, Yue Xiao ${ }^{\circledR}$, Member, IEEE, \\ Ming Xiao ${ }^{\circledR}$, Senior Member, IEEE, and Wei Xiang ${ }^{\circledR}$, Senior Member, IEEE
}

\begin{abstract}
This paper proposes a precoded optical space-domain index modulation scheme for indoor visible light communications, which is based on the optimization of the minimum Euclidean distance of optical spatial modulation (OSM) with real-valued modulation constellations. We find that the precoding matrix design can be formulated as a non-convex quadratically constrained quadratic program (QCQP), whose solution is generally intractable. To tackle this problem, we first consider the case of two optical transmit antennas $\left(N_{t}=2\right)$ in the precoded OSM and derive a closed-form solution for arbitrary $M$-order pulse amplitude modulation (PAM). Based on the derived solutions and the error vector reduction method, we then propose a low-complexity iterative (LCI) algorithm to identify the precoding matrix for the setup $N_{t}>2$. To strike a flexible complexity-BER (bit error rate) tradeoff, we propose a successive convex approximation (SCA)-assisted matrix-based optimization method to transform the non-convex QCQP problem into a series of linear convex subproblems, which can be solved by low-complexity solvers. Simulation results show that these proposed algorithms are capable of substantially improving the system error performance compared with conventional OSM systems. Besides, a symbol-based SCA algorithm is introduced and it is shown to outperform the matrix-based SCA and the suboptimal LCI algorithm in terms of the BER.
\end{abstract}

Index Terms-Convex optimization, index modulation, precoding, spatial modulation, visible light communication.

\section{INTRODUCTION}

$\mathbf{W}$ ITH the application and development of $5 \mathrm{G}$ technology, it is urgent to deal with the issue of ever

Manuscript received March 4, 2020; revised September 4, 2020; accepted November 18, 2020. Date of publication December 1, 2020; date of current version April 16, 2021. This work is supported by the National Science Foundation of China under Grant number U19B2014, and the National Science Foundation of China under Grant number 61876033. The associate editor coordinating the review of this article and approving it for publication was H. Elgala. (Corresponding author: Ping Yang.)

Yongyang $\mathrm{Li}$ and Ping Yang are with the National Key Laboratory of Science and Technology on Communications, University of Electronic Science and Technology of China, Chengdu 611731, China, and also with the State Key Laboratory of Integrated Services Networks, Xidian University, Xi'an 710071, China (e-mail: 851465089@qq.com; yang.ping@uestc.edu.cn).

Marco Di Renzo is with Université Paris-Saclay, CNRS, CentraleSupélec, Laboratoire des Signaux et Systèmes, 91192 Gif-sur-Yvette, France (e-mail: marco.di-renzo@universite-paris-saclay.fr).

Yue Xiao is with the National Key Laboratory of Science and Technology on Communications, University of Electronic Science and Technology of China, Chengdu 611731, China (e-mail: xiaoyue@uestc.edu.cn).

Ming Xiao is with the Information Science and Engineering (ISE) Department, School of Electrical Engineering, KTH Royal Institute of Technology, 11428 Stockholm, Sweden (e-mail: mingx@kth.se).

Wei Xiang is with the School of Engineering and Mathematical Sciences, La Trobe University, Melbourne, VIC 3086, Australia (e-mail: w.xiang@latrobe.edu.au). increasing data communication demands. The visible light band is a promising frequency band for communications since it encompasses a large spectrum of license-free bandwidth that can solve the lack of available spectrum in the low frequency band [1]. In general, visible light communications (VLC) is a powerful complement for designing high-capacity cellular networks and it brings some attractive advantages, such as the inherent high security, low cost, and low inter-cell interference [2]. VLC can complement WiFi, since Internet users spend most of their time indoors (more than $80 \%$ of the time). As shown in some recent papers, such as [3] and [4], the untapped potential of VLC makes it a very promising candidate for future $6 \mathrm{G}$ networks.

Different from classical radio frequency (RF) communication systems, light emitting diodes (LEDs) are employed in VLC. This leads to the use of intensity modulation and direct detection (IM/DD) techniques for most front ends of VLC systems [5], [6]. The transmit information is conveyed in the changes of the intensity of the emitted data, and the receiver detects the electrical signal that is proportional to the intensity of the detected light. The use of IM/DD methods ensures that the transmit signal is real and non-negative [6], [7]. As a result, selecting suitable modulation methods with the objective of achieving high transmission rates and high power efficiency becomes a research problem in its own right.

Against this background, some conventional modulation techniques, such as the on-off keying (OOK) modulation, pulse position modulation (PPM) and pulse amplitude modulation (PAM) were proposed for VLC systems [8] and [9]. Another solution is to increase the dimension of conventional modulations by utilizing RF-based multipleinput multiple-output (MIMO) techniques. For example, in [10]-[13] the multiplexing and diversity concepts of MIMO for RF communications were extended to VLC systems, with the goal of improving the spectral efficiency and error performance. In [14], a high-dimensional real-valued modulation scheme was proposed for VLC systems, which transmits the positive real, negative real, positive imaginary, and negative imaginary parts of the modulated symbols according to the position of the antennas of the transmitter so as to overcome the limitation that the VLC front-end cannot transmit complex-modulated symbols. Moreover, many other promising constellation designs, such as the optimal constellation design, the space-collaborative constellation design, the systematic method based design, as well as the channel-adaptive space-collaborative constellation design were proposed to exploit the spatial degrees of freedom of multiple LEDs from different perspectives [15], [16]. 
In these designs, the use of a large number of LEDs may result in increasing the complexity of transmit signal generation and detection.

In this context, optical spatial modulation (OSM) is an emerging technique that offers an attractive low-complexity and high-rate design option for VLC-MIMO systems [17], [18]. In OSM, multiple spatially separated light sources are considered and only one of the light sources is activated at each channel use [19]. The activated LED emits the real and positive constellation symbols and thus OSM retains the benefits of multi-stream VLC-MIMO [20]. The potential benefits of VLC-OSM over conventional VLC-MIMO techniques have been validated not only via numerical simulations but also by laboratory experiments [21]-[24]. More recently, some variants of OSM have been proposed to facilitate the design of intensity modulation in various VLC scenarios. For example, in [25] a generalized OSM scheme for orthogonal frequency division multiplexing (OFDM)-based VLC systems was proposed, and two novel receivers were designed for frequency-flat and frequency-selective channels. The authors of [26] introduced an adaptive spatial modulation (ASM) scheme for VLC systems, and two algorithms were proposed to optimize the modulation order given a fixed spectral efficiency. In [27], the authors proposed receiver-oriented OSM to mitigate the optical channel correlation among multiple LEDs. In [28], a bipolar PAM-based OSM scheme was developed, where the information on the polarity of PAM symbols was conveyed via the indexes of the LEDs.

In order to further exploit the available spatial-domain degrees of freedom that are offered by VLC-MIMO channels, linear transmit precoding (TPC) techniques can be adopted. Indeed, TPC has been widely investigated in the context of conventional MIMO-aided VLC systems. Specifically, one simple way to design the precoder for MIMO-aided VLC systems is via channel matrix inversion, e.g., the zero-forcing precoding and minimum mean square error (MMSE) precoding [29]. Furthermore, a joint precoder and equalizer design for spatial multiplexing VLC systems was proposed in [30], which was formulated as an optimization problem and solved by an iterative updating method. However, since only a single transmit antenna is activated at each time slot in OSM, TPC methods for conventional VLC-MIMO are not directly suitable for OSM systems. Moreover, it is worth noting that although there exist some TPC schemes designed for RF-based spatial modulation MIMO (SM-MIMO) systems [31], the precoder design in OSM requires a special consideration, since the VLC constellation symbols have to be positive and real-valued.

More specifically, the precoder design for VLC-OSM systems remains an open problem. In previous works, only some initial preprocessing methods at the transmitter are considered for OSM. For example, a symbol set selection algorithm was proposed in [32] for generalized space shift keying (GSSK) VLC, and hardware experiments were carried out to verify its benefits. In [33], a multi-user precoding based block diagonalization was investigated for OSM in order to achieve robust link and rate performance. In [34], a novel iterative combinatorial symbol design algorithm for GSM was proposed, which improves the error performance of a random symbol set by iteratively adding and removing symbols. It is shown that the proposed scheme is capable of providing a considerable bit error rate (BER) gain over other existing symbol design algorithms. Moreover, in order to improve the secrecy performance, the authors of [35] derived bounds for the secrecy rate of indoor SM-VLC systems, and proposed channel adaptive selection and greedy selection schemes to select the active transmitter. A constellation selection algorithm was proposed for OSM to minimize the average transmit power in [36], while a channel-adaptive bit mapping rule was proposed for OSM to provide more design flexibility in [37]. More recently, based on the results in [37], the bit-to-symbol mapping problem in OSM was further investigated in [38] and [39].

Among the promising design alternatives, the TPC technique constitutes an attractive transmit preprocessing alternative. However, to the best of our knowledge, the potential benefits of TPC have not been fully investigated in OSM systems.

Against the above background, the main contributions of this paper are as follows.

- We propose a precoded OSM (POSM) for indoor VLC, which is capable of effectively supporting both data transmission and lighting requirements. Compared to the conventional precoding matrix design for RF-aided SM-MIMO, we design the precoder through optimizing the minimum Euclidean distance for real-valued modulation constellations, which ensures that the precoded vectors are real and positive as they represent light intensities;

- We derive the closed-form optimal solution to the real-valued precoding matrix for POSM systems when the number of transmit antennas is $N_{t}=2$ for arbitrary $M$-order PAM and arbitrary numbers of receive antennas. In our design, the complexity of calculating the closed-form solution is reduced by shrinking the space of error vectors. Specifically, we divide the Euclidean distance into three subsets, remove the collinear vector, and consider only the minimum values of some subsets when calculating the solution. Furthermore, we extend this method to the setup $N_{t}>2$;

- To strike a flexible complexity-BER tradeoff, we convert the original non-convex precoding optimization problem into an approximate convex problem by using the successive convex approximation (SCA) method. Simulation results show that the error performance of the precoding matrix derived by the proposed algorithm is close to that of the closed-form solution. Besides matrix-based SCA POSM, we also investigate symbol-based SCA precoding POSM, where the transmit symbols and the channel state information are jointly considered. It is found that this joint design helps significantly improve the BER.

Notation: Boldface uppercase letters denote matrices, while boldface lowercase letters represent vectors. The superscripts $(\cdot)^{T}$ and $(\cdot)^{H}$ denote transpose and conjugate transpose oper- 


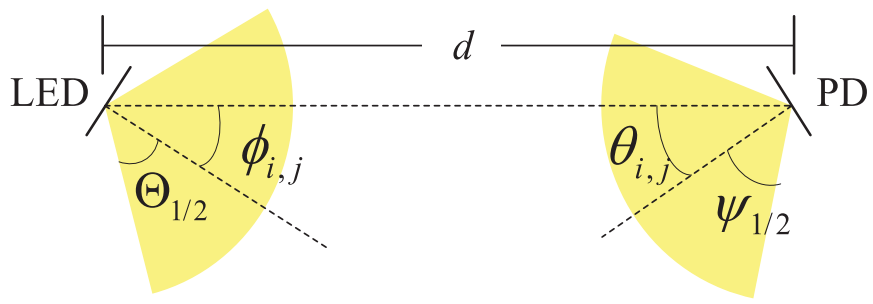

Fig. 1. Geometric representation of the channel parameters.

ators, respectively. $\|\cdot\|$ is the Frobenius norm and $\operatorname{tr}(\cdot)$ is the trace of a matrix; The real number field is denoted by $\mathbb{R}$. abs $(\cdot)$ is the absolute operator, $\mathbf{I}_{N}$ stands for the identity matrix, while the operator $\operatorname{diag}\{\cdot\}$ applied to a length$i$ vector returns an $i \times i$ square matrix with the vector elements along the diagonal. Furthermore, $\odot$ represents the Hadamard product, $Q(\cdot)$ denotes the Q-function, and $C_{N}^{k}$ denotes the number of combinations of selecting $k$ out of $N$ numbers. $(\mathbf{A})_{k l}$ denotes the element in the $k$ th row and the $l$ th column of matrix $\mathbf{A}, E(\cdot)$ denotes the expectation, and $\operatorname{Re}\{\mathbf{x}\}$ and $\operatorname{Im}\{\mathbf{x}\}$ denote the real and imaginary parts of $\mathbf{x}$, respectively.

\section{System Model of the Proposed Precoded OSM}

\section{A. Channel Model}

Consider an OSM system with $N_{t}$ optical transmit antennas (TAs) and $N_{r}$ photodetectors (PDs). In general, the channel components of VLC contain two types of links, namely the direct point-to-point line-of-sight (LOS) link and the diffuse link caused by reflections [6]. As shown in [1], [2], the LOS link component is usually much stronger than its diffuse counterpart. Hence, similar to the channel model given in [21], [30], in this paper we only consider the LOS link, and the channel impulse response between the $j$ th TA and the $i$ th PD is modeled as

$$
h_{i j}=\left\{\begin{array}{cc}
\frac{(m+1) A}{2 \pi d_{i, j}^{2}} \cos ^{m} \phi_{i, j} \cos \theta_{i, j} & 0 \leq \theta_{i, j} \leq \psi_{1 / 2}, \\
0 & \theta_{i, j}>\psi_{1 / 2}
\end{array}\right.
$$

where $m=-\frac{\ln 2}{\ln \left(\cos \left(\Theta_{1 / 2}\right)\right)}$ is the order of Lambertian emission and $\Theta_{1 / 2}$ is the transmitter semi-angle. Moreover, as shown in Fig. $1, \phi_{i, j}$ in (1) is the angle of emergence with respect to the transmitter axis of the TA for the channel between the $j$ th TA and the $i$ th $\mathrm{PD}, \theta$ is the angle of incidence relative to the receiver axis for the channel between the $j$ th TA and the $i$ th $\mathrm{PD}, d_{i, j}$ is the distance between the $j$ th TA and the $i$ th PD, $A$ is the detector area of the PD, while $\psi_{1 / 2}$ denotes the field-of-view semi-angle of the receiver.

Based on the channel tap model given in (1), the VLC MIMO channel $\mathbf{H}$ can be expressed as

$$
\mathbf{H}=\left[\begin{array}{cccc}
h_{11} & h_{12} & \cdots & h_{1 N_{t}} \\
h_{21} & h_{21} & \cdots & h_{2 N_{t}} \\
\vdots & \vdots & \ddots & \vdots \\
h_{N_{r} 1} & h_{N_{r} 2} & \cdots & h_{N_{r} N_{t}}
\end{array}\right]
$$

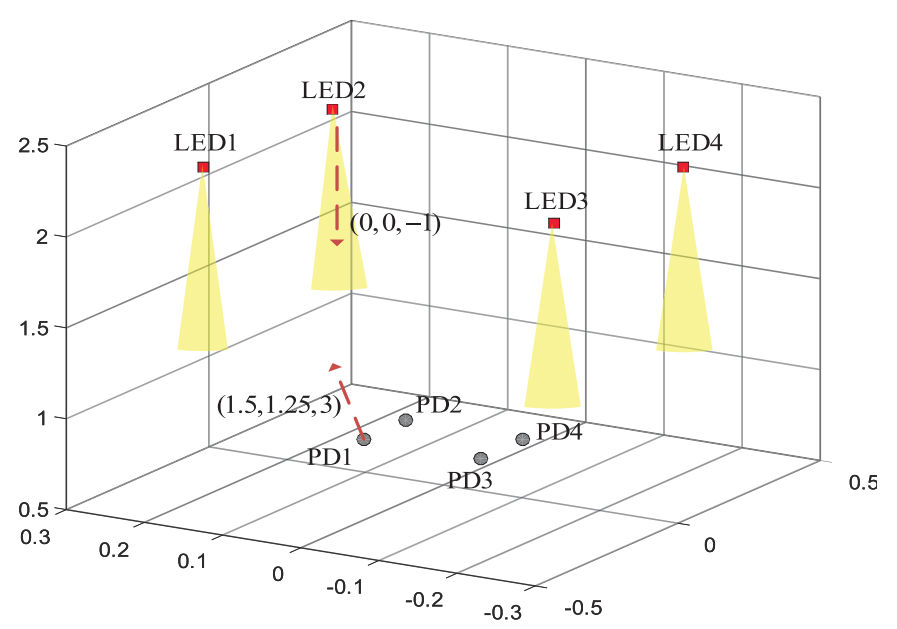

Fig. 2. Example of $4 \times 4$ VLC MIMO indoor deployment.

Fig. 2 shows an example of the possible positions of the TAs and PDs in the VLC MIMO system. Referring to [22], adjusting the angle of the receiver can reduce the correlation of the channel, so we skew the receiver axis of the PDs.

\section{B. Transmitter and Receiver}

This section describes the transmitter model and the detection algorithm for the $\left(N_{t} \times N_{r}\right)$-element precoded OSM system. As illustrated in Fig. 3, the bitstream is first modulated by using the SM concept, where one out of $N_{t}$ TAs is activated in each time slot, and then one PAM constellation symbol is transmitted by the activated antenna. It is worth noting that VLC MIMO systems employ the IM/DD technique and hence the transmit signals need to be non-negative and realvalued. Since PAM modulation is easy to implement and also a common modulation mode in most VLC systems, we employ PAM modulation for the OSM system under consideration. ${ }^{1}$

Specifically, in the proposed precoded OSM scheme, the bit stream is divided into two parts in each time slot, where $m_{1}=$ $\log _{2}\left(N_{t}\right)$ bits are used for selecting a unique TA, while $m_{2}=$ $\log _{2}(M)$ bits are mapped into an $M$-PAM constellation point. After this SM-type mapping, the resulting modulated signal vector $\mathbf{s} \in \mathbb{R}^{N_{t} \times 1}$ is given by

$$
\begin{gathered}
\mathbf{s}=\mathbf{e}_{n} s_{m}=[0,0, \cdots, \\
\left.s_{m}, \cdots, 0,0\right] \in \mathbb{S}, \\
\uparrow n \text {th term }
\end{gathered}
$$

where $s_{m} \in \mathbb{R}, m=1,2, \cdots M$ is the $m$ th symbol in the $M$ PAM constellation points, and $\mathbf{e}_{n}$ is the $N_{t}$-dimensional standard basis vector used to indicate the $n$th active antenna. $\mathbb{S}$ is the set of all transmit symbols after OSM mapping. Note that the PAM modulation symbols $s_{m}$ are bounded by

$$
-b \leq s_{m} \leq b, \forall m=1 \ldots M .
$$

For a specific PAM scheme, the boundary value $b$ is assumed to be the maximum amplitude of PAM symbols, given by

\footnotetext{
${ }^{1}$ Note that we do not impose specific restrictions on the underlying modulation symbols for the proposed systems. Besides real-valued modulation schemes, e.g., PAM and SSK, our proposed algorithms can also be extended to complex-valued modulation schemes. For example, we can consider the application of the asymmetrically clipped optical OFDM (ACO-OFDM) technique to the OSM system with PSK and QAM modulation, so as to ensure that the modulated signal is real.
} 


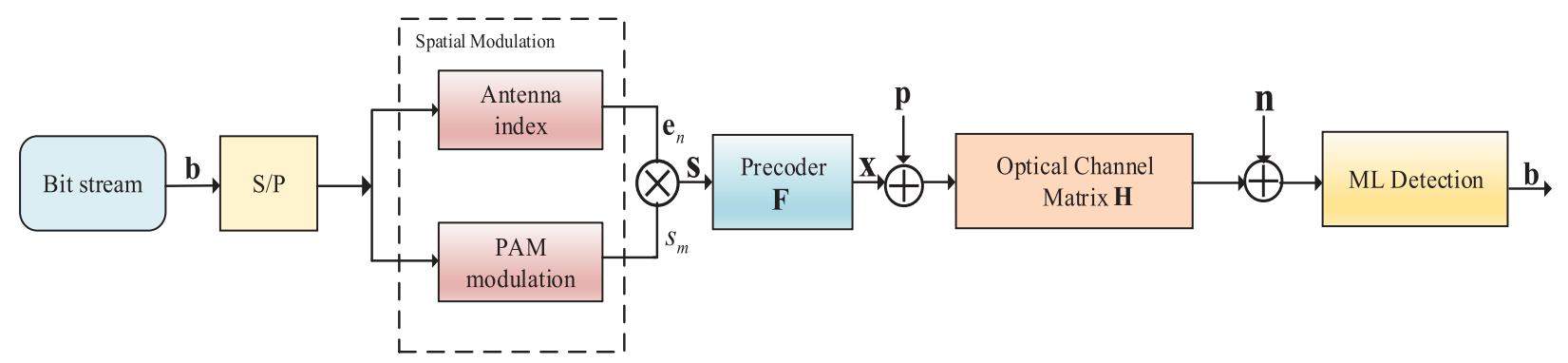

Fig. 3. The transceiver model of the precoded OSM system.

$(M-1) / \sqrt{E_{\mathrm{s}}}$, where $E_{\mathrm{s}}=(M-1)(M+1) / 3$ is the average power of the unnormalized signal vector $\mathbf{s}$. Before the data vector is sent to the optical channels, it is precoded by a precoding matrix and a direct current (DC) bias vector $\mathbf{p}=$ $\left[p_{1}, p_{2}, \cdots, p_{N_{t}}\right]^{T}$ is added to ensure that the final signal is non-negative. Specifically, the precoding matrix $\mathbf{F} \in \mathbb{R}^{N_{t} \times N_{t}}$ is

$$
\mathbf{F}=\operatorname{diag}\left\{f_{1,1}, f_{2,2}, \cdots, f_{N_{t}, N_{t}}\right\} .
$$

The diagonal structure of the precoding matrix $\mathbf{F}$ guarantees that the precoded vectors Fs have a single non-zero element, hence the benefits of conventional OSM are preserved, such as low complexity and signal sparsity.

The elements of the precoding matrix $f_{i, j}\left(f_{i, j}=0, i \neq\right.$ $j$ ) satisfy the following constraint of the total transmit power $P_{T}$

$$
\operatorname{tr}\left(\mathbf{F}^{T} \mathbf{F}\right)=\sum_{i=1}^{N_{t}}\left|f_{i, i}\right|^{2} \leq P_{T} .
$$

Moreover, in a precoded OSM system the DC bias vector $\mathbf{p}$ can be adjusted to achieve a specific illumination level in order to support dimming or brightness control. In order to guarantee that the transmitted OSM vector is positive, the vector $\mathbf{p}$ can be expressed as

$$
\begin{aligned}
& \left\{\begin{array}{c}
x_{i}=\sum_{j=1}^{N_{t}} s_{j} f_{i, j} \geq-p_{i} \\
-b_{m} \leq s_{m} \leq b_{m}
\end{array} \Rightarrow\right. \\
& \left\{\begin{array}{c}
-\sum_{j=1}^{N_{t}} b_{j}\left|f_{i, j}\right| \leq x_{i} \leq \sum_{j=1}^{N_{t}} b_{j}\left|f_{i, j}\right| \\
-p_{i} \leq x_{i}
\end{array}\right.
\end{aligned}
$$

For the sake of simplification, we can reformulate the constraint in (8) into its corresponding matrix form as follows

$$
\operatorname{abs}(\mathbf{F}) \mathbf{b} \leq \mathbf{p}
$$

where $b_{i}$ is the $i$ th element of vector $\mathbf{b}$. The elements in $\mathbf{b}$ and $\mathbf{p}$ are assumed equal in this paper to simplify the calculations. We assume that the channel response of VLC-MIMO in each transmission period is independent. Then, the signal vector observed at the receiver is given by

$$
\mathbf{y}=\mathbf{H}(\mathbf{F s}+\mathbf{p})+\mathbf{n}
$$

where $\mathbf{n}$ is the $\left(N_{r} \times 1\right)$-element noise vector. We further assume $E\left[\mathbf{n n}^{H}\right]=N_{0} \mathbf{I}_{N_{r}}$ and $E\left[\mathbf{s n}^{H}\right]=\mathbf{0}_{N_{t} \times N_{t}}$. The elements of the noise vector $\mathbf{n}$ are complex Gaussian random variables obeying $\mathcal{C N}\left(0, N_{0}\right)$. The receiver performs maximum-likelihood (ML) detection over all possible OSM symbols $\mathbf{s} \in \mathbb{R}^{N_{t} \times 1}$ for retrieving the transmit symbols.

\section{Algorithms for Precoding Matrix Design}

In this section, we optimize the performance of the precoded OSM system by maximizing the minimum Euclidean distance of real-valued modulation constellations. The precoder design also ensures that the resulting transmit vectors are real and positive. Combined with the constrains of the precoding matrix derived in Section II, we establish the optimization problem and derive solutions for various VLC MIMO configurations.

\section{A. Performance Metric and Optimization Criterion}

As introduced in Section II, the receiver employs the ML criterion to detect the TA index as well as the PAM symbol. The overall average pairwise error probability (PEP) conditioned on $\mathbf{H F}$ is given by

$$
\begin{aligned}
P_{\mathrm{PEP}}(\mathbf{H F})_{e} & \leq \frac{1}{L} \sum_{i=1}^{L} \sum_{j=1, j \neq i}^{L} Q\left(\sqrt{\frac{\left\|\mathbf{H F}\left(\mathbf{s}_{i}-\mathbf{s}_{j}\right)\right\|^{2}}{2 N_{0}}}\right) \\
& \leq \frac{1}{L} \times L(L-1) Q\left(\sqrt{\frac{d_{\text {min }}}{2 N_{0}}}\right)=(L-1) Q\left(\sqrt{\frac{d_{\text {min }}}{2 N_{0}}}\right)
\end{aligned}
$$

where $d_{\text {min }}=\min _{i \neq j}\left\|\mathbf{H F}\left(\mathbf{s}_{i}-\mathbf{s}_{j}\right)\right\|^{2}$ is the minimum Euclidean distance (i.e., the free distance) of the precoded OSM vectors, and $L=M N_{t}$. Thanks to the monotonicity of function $Q(\cdot)$, the error performance can be improved by maximizing the free distance $d_{\min }$. Combing the constraints in (6), (9) and the objective function in (11), the precoding matrix design problem is formulated as follows

$$
\begin{array}{ll}
\underset{\mathbf{F}}{\arg } \max \left(\min _{i \neq j}\left\|\mathbf{H F}\left(\mathbf{s}_{i}-\mathbf{s}_{j}\right)\right\|^{2}\right) \\
\text { s.t. } & \operatorname{abs}(\mathbf{F}) \mathbf{b} \leq \mathbf{p}, \\
& \operatorname{tr}\left(\mathbf{F F}^{T}\right) \leq P_{T} .
\end{array}
$$

In the following subsections, we first derive the closed-form optimal solution for the real-valued precoding matrix for 2PAM modulated precoded OSM systems with two antennas at the transmitter, and then extend the analysis to arbitrary $M$-order PAM. Moreover, a low-complexity iterative optimization solver based on the derived closed-form solution and an approximate SCA method are proposed for more general MIMO configurations. 


\section{B. Optimal Precoding Matrix for 2PAM Modulated OSM With $N_{t}=2$}

In a 2PAM modulated OSM system with two antennas at the transmitter, we can obtain all the Euclidean distances $d=\left\|\mathbf{H F}\left(\mathbf{s}_{i}-\mathbf{s}_{j}\right)\right\|^{2}, i \neq j$ via an exhaustive search, because the OSM constellation set $\mathbb{S}$ has only a few elements. Let the precoding matrix be $\mathbf{F}=\operatorname{diag}\left\{f_{1,1}, f_{2,2}\right\}=\operatorname{diag}\left\{f_{1}, f_{2}\right\}$. Moreover, the $\left(N_{r} \times 2\right)$-element channel matrix $\mathbf{H}$ can be decomposed into two column vectors $\mathbf{h}_{1}$ and $\mathbf{h}_{2}$. Based on these expressions, all legitimate distances based on (11) are given by

$$
\left\{\begin{aligned}
d_{1} & =\left\|\mathbf{H F}[2,0]^{\mathrm{T}}\right\|^{2}=4 f_{1}^{2}\left\|\mathbf{h}_{1}\right\|^{2}, \\
d_{2} & =\left\|\mathbf{H F}[0,2]^{\mathrm{T}}\right\|^{2}=4 f_{2}^{2}\left\|\mathbf{h}_{2}\right\|^{2}, \\
d_{3} & =\left\|\mathbf{H F}[1,-1]^{\mathrm{T}}\right\|^{2}=\left\|f_{1} \mathbf{h}_{1}-f_{2} \mathbf{h}_{2}\right\|^{2}, \\
d_{4} & =\left\|\mathbf{H F}[1,1]^{\mathrm{T}}\right\|^{2}=\left\|f_{1} \mathbf{h}_{1}+f_{2} \mathbf{h}_{2}\right\|^{2},
\end{aligned}\right.
$$

where $[2,0]^{T},[0,2]^{T},[1,-1]^{T}$ and $[1,1]^{T}$ are the remaining possible error vectors $\mathbf{s}_{i}-\mathbf{s}_{j}$ after removing the collinear vectors for simplifying the calculation. Assuming that $f_{1}$ is a variable, $f_{2}$ can be expressed as $f_{2}=\sqrt{P_{T}-f_{1}^{2}}$. Based on (12) and (13), deriving the optimal precoder solution of OSM for the 2PAM modulated $2 \times N_{r}$ OSM system is equivalent to finding the closed-form solution of $\mathbf{F}$ which can maximize the minimum distance among the four distances $d_{i}, i=1,2,3,4$. The problem can be formulated as follows

$$
\begin{array}{cc}
\max _{f_{1}, f_{2}} \min \left\{d_{1}, d_{2}, d_{3}, d_{4}\right\} \\
\text { s.t. } & f_{1}{ }^{2} \leq(p / b)^{2}, \\
& f_{2}{ }^{2} \leq(p / b)^{2} \\
& f_{1}^{2}+f_{2}{ }^{2}=P_{T} .
\end{array}
$$

To obtain the specific precoding matrix $\mathbf{F}$ that is able to maximize the distance $d_{\min }$, the parameters $f_{1}$ and $f_{2}$ (with $f_{2}$ depending on $f_{1}$ ) have to be computed. To this end, an intuitive yet effective method is to calculate the intersections of the four distance functions with respect to the variable $f_{1}$, and then find the suitable intersection point by comparing the minimum distance values. As for the optimization problem given in (14), the solution that maximizes $d_{\min }$ is at the intersection of the four curves that correspond to the receive distances $d_{1}$ to $d_{4}$.

As can be seen from (14), $d_{1}, d_{2} d_{3}$ and $d_{4}$ are functions of the parameter $f_{1}^{2}$, and there are five possible intersections between two arbitrary two distances, hence the five possible solutions of the precoding matrix parameters $f_{1}^{(i)}$ and $f_{2}^{(i)}, i=$ $1, \cdots, 5$ are given by

$$
\begin{aligned}
& \left\{\begin{array}{l}
f_{1}^{(1)}=\sqrt{g P_{T} /(a+g)} \\
f_{2}^{(1)}=\sqrt{a P_{T} /(a+g)},
\end{array}\right. \\
& \left\{\begin{array}{l}
f_{1}^{(2)}=\sqrt{\frac{c^{2}+6 a c-c \sqrt{c^{2}+12 a g}}{18 a^{2}+c^{2}+6 a g-c \sqrt{c^{2}+12 a g}} P_{T}} \\
f_{2}^{(2)}=\sqrt{\frac{18 a^{2}}{18 a^{2}+c^{2}+6 a g-c \sqrt{c^{2}+12 a g}} P_{T}}
\end{array}\right.
\end{aligned}
$$

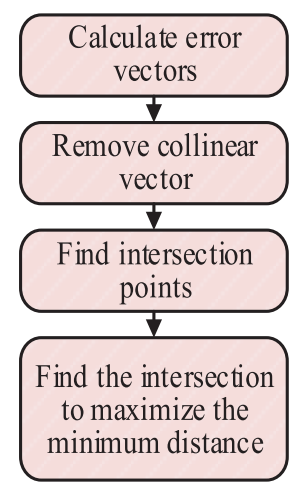

Fig. 4. Flow chart to find a closed-form solution to the optimization problem for OSM systems.

$$
\begin{aligned}
& \left\{\begin{array}{l}
f_{1}^{(3)}=\sqrt{\frac{c^{2}+6 a c+c \sqrt{c^{2}+12 a g}}{18 a^{2}+c^{2}+6 a g+c \sqrt{c^{2}+12 a g}} P_{T}} \\
f_{2}^{(3)}=\sqrt{\frac{18 a^{2}}{18 a^{2}+c^{2}+6 a g+c \sqrt{c^{2}+12 a g}} P_{T}},
\end{array}\right. \\
& \left\{\begin{array}{l}
f_{1}^{(4)}=\sqrt{\frac{c^{2}+6 a c+c \sqrt{c^{2}+12 a g}}{2 a^{2}+c^{2}+6 a g+c \sqrt{c^{2}+12 a g}} P_{T}} \\
f_{2}^{(4)}=\sqrt{\frac{2 a^{2}}{2 a^{2}+c^{2}+6 a g+c \sqrt{c^{2}+12 a g}} P_{T}},
\end{array}\right. \\
& \left\{\begin{array}{l}
f_{1}^{(5)}=\sqrt{\frac{c^{2}+6 a c-c \sqrt{c^{2}+12 a g}}{2 a^{2}+c^{2}+6 a g-c \sqrt{c^{2}+12 a g}} P_{T}} \\
f_{2}^{(5)}=\sqrt{\frac{2 a^{2}}{2 a^{2}+c^{2}+6 a g-c \sqrt{c^{2}+12 a g}} P_{T}},
\end{array}\right.
\end{aligned}
$$

where $f_{1}^{(i)}, i=1, \cdots, 5$ are the TPC weights assigned to the first TA of the $i$ th intersection. Here, we have $a=\left\|\mathbf{h}_{1}\right\|^{2}$, $g=\left\|\mathbf{h}_{2}\right\|^{2}$, and $c=2 \mathbf{h}_{1}{ }^{T} \mathbf{h}_{2}$. Based on (14)-(18), we select the one providing the maximum $d_{\min }$ as the final solution.

\section{Optimal Precoding Matrix for $2 \times N_{r}$ OSM With Arbitrary PAM}

Inspired by the derivation of the closed-form solution in the previous subsection, we further derive the solution to the TPC optimization problem of OSM systems for higher-order modulation, i.e., $M>2$. Calculating $N_{t} M\left(N_{t} M-1\right)$ error vectors is impractical for high-rate scenarios as the modulation order increases. To tackle this challenge, Fig. 4 depicts a flow chart for deriving the closed-form solution by using the error vector reduction method. Specifically, since the OSM symbols $\mathbf{s}_{i}\left(\mathbf{s}_{i} \in \mathbb{S}\right)$ only have a single non-zero element, the error vectors $\mathbf{e}_{i j}=\mathbf{s}_{i}-\mathbf{s}_{j}, i \neq j$ can be classified into two types, namely the error vectors with only a single non-zero element, and those with two non-zero elements. The received distances $\left\|\mathbf{H F}\left(\mathbf{s}_{i}-\mathbf{s}_{j}\right)\right\|^{2}$ can be grouped into two sets $\mathbb{D}_{2}$ and $\mathbb{D}_{2}$ given as follows

$$
\begin{aligned}
& \mathbb{D}_{1}=\left\{f_{i}^{2}\left\|\mathbf{h}_{i}\right\|^{2}\left(s_{l}-s_{\hat{\imath}}\right)^{2}, l \neq \widehat{l}, i=1,2\right\}, \\
& \mathbb{D}_{2}=\left\{\left\|f_{1} s_{l} \mathbf{h}_{1}-f_{2} s_{\hat{l}} \mathbf{h}_{2}\right\|^{2}, l, \widehat{l}=1,2, \cdots M\right\},
\end{aligned}
$$

Since the distance intervals of the PAM symbols are equal, the smallest element in set $\mathbb{D}_{1}$ is the minimum value between $d_{M-\text { PAM }}^{2} f_{1}^{2}\left\|\mathbf{h}_{1}\right\|^{2}$ and $d_{M-\text { PAM }}^{2} f_{2}^{2}\left\|\mathbf{h}_{2}\right\|^{2}$, where $d_{M-\mathrm{PAM}}$ is the distance between two adjacent $M$ PAM constellation points. Therefore, we only need to consider the intersections between $d_{M-P A M}^{2} f_{1}^{2}\left\|\mathbf{h}_{1}\right\|^{2}$ and 
$d_{M-\text { PAM }}^{2} f_{2}{ }^{2}\left\|\mathbf{h}_{2}\right\|^{2}, d_{M-P A M}^{2} f_{1}^{2}\left\|\mathbf{h}_{1}\right\|^{2}$ and the elements in the set $\mathbb{D}_{2}, d_{M-\text { PAM }}^{2} f_{2}{ }^{2}\left\|\mathbf{h}_{2}\right\|^{2}$ and the elements in the set $\mathbb{D}_{2}$, as well as the intersections between two arbitrary elements in $\mathbb{D}_{2}$.

Specifically, the candidate intersection point of $d_{M-\text { PAM }}^{2} f_{1}^{2}\left\|\mathbf{h}_{1}\right\|^{2}$ and $d_{M-\text { PAM }}^{2} f_{2}{ }^{2}\left\|\mathbf{h}_{2}\right\|^{2}$ needs to satisfy

$$
d_{M-\mathrm{PAM}}^{2} f_{1}^{2}\left\|\mathbf{h}_{1}\right\|^{2}=d_{M-\mathrm{PAM}}^{2}{f_{2}}^{2}\left\|\mathbf{h}_{2}\right\|^{2} .
$$

The corresponding solution $\left(f_{1}, f_{2}\right)$ to $(21)$ is given by

$$
\left\{\begin{array}{l}
f_{1}=\sqrt{g P_{T} /(a+g)} \\
f_{2}=\sqrt{a P_{T} /(a+g)}
\end{array} .\right.
$$

To obtain the intersections between $d_{M-\mathrm{PAM}}^{2} f_{1}^{2}\left\|\mathbf{h}_{1}\right\|^{2}$ and the elements of $\mathbb{D}_{2}, f_{1}$ and $f_{2}$ need to satisfy the following constraints

$$
\left\{\begin{array}{l}
d_{M-\mathrm{PAM}}^{2} f_{1}^{2}\left\|\mathbf{h}_{1}\right\|^{2}=\left\|f_{1} s_{l} \mathbf{h}_{1}-f_{2} s_{l} \mathbf{h}_{2}\right\|^{2} \\
f_{1}^{2}+f_{2}^{2}=P_{T}
\end{array}\right.
$$

Since only real and positive-valued solutions to (23) are considered for OSM systems, the intersection point can be expressed as

$$
\left\{\begin{array}{c}
f_{2}=\sqrt{P_{T} /\left(A^{2}+1\right)} \\
f_{1}=A \sqrt{P_{T} /\left(A^{2}+1\right)},
\end{array}\right.
$$

where $A=\frac{-c s_{l} s_{\hat{l}}+s_{\hat{l}} \sqrt{c^{2} s_{l}^{2}+4 a g\left(4-s_{l}^{2}\right)}}{2 a\left(4-s_{l}^{2}\right)}$. It is unnecessary to consider all the elements in $\mathbb{D}_{2}$ when calculating the legitimate intersection points. It is worth noting that half of the $M^{2}$ symbol pairs $\left(s_{a}, s_{b}\right)$ selected to calculate the distances in $\mathbb{D}_{2}$ are collinear. As a result, the computational complexity can be reduced from the order of $M^{2}$ to that of $M^{2} / 2$. Similarly, the solutions $f_{1}$ and $f_{2}$ in the third case can be obtained by finding the intersection points between $d_{M-\mathrm{PAM}}^{2}{f_{2}}^{2}\left\|\mathbf{h}_{2}\right\|^{2}$ and the $M^{2} / 2$ non-collinear elements in the set $\mathbb{D}_{2}$, which can be expressed as

$$
\left\{\begin{array}{c}
f_{1}=\sqrt{P_{T} /\left(B^{2}+1\right)} \\
f_{2}=B \sqrt{P_{T} /\left(B^{2}+1\right)}
\end{array}\right.
$$

where $B=\frac{-c s_{l} s_{\widehat{l}}+s_{l} \sqrt{c^{2} s_{\widehat{l}}^{2}+4 a g\left(4-s_{\widehat{l}}^{2}\right)}}{2 g\left(4-s_{\widehat{l}}^{2}\right)}$.

Furthermore, we can also derive the possible intersections between arbitrary received distances in the set $\mathbb{D}_{2}$. Note that there are $C_{M^{2} / 2}^{2}$ distance pairs, hence the $C_{M^{2} / 2}^{2}$ legitimate intersections need to satisfy the following constrains

$$
\left\{\begin{array}{l}
\left\|f_{1} s_{a} \mathbf{h}_{1}-f_{2} s_{b} \mathbf{h}_{2}\right\|^{2}=\left\|f_{1} s_{c} \mathbf{h}_{1}-f_{2} s_{d} \mathbf{h}_{2}\right\|^{2} \\
f_{1}^{2}+f_{2}^{2}=P_{T}
\end{array}\right.
$$

where $s_{a}, s_{b}, s_{c}$, and $s_{d}$ denote the PAM symbols. According to (26), the solutions are given by

$$
\left\{\begin{array}{l}
f_{2}=\sqrt{P_{T} /\left(C^{2}+1\right)} \\
f_{1}=C \sqrt{P_{T} /\left(C^{2}+1\right)}
\end{array}\right.
$$

where $\quad C=\left(\frac{\sqrt{\left(s_{c} s_{d}-s_{a} s_{b}\right)^{2} c^{2}-4 a g\left(s_{a}^{2}-s_{c}^{2}\right)\left(s_{b}^{2}-s_{d}^{2}\right)}}{2 a\left(s_{a}^{2}-s_{c}^{2}\right)}+\right.$ $\left.\frac{-c\left(s_{c} s_{d}-s_{a} s_{b}\right)}{2 a\left(s_{a}^{2}-s_{c}^{2}\right)}\right)$. The minimum distances of these candidate solutions given in (22), (24) and (27) are generated and from them the optimal solution having the largest $d_{\min }$ is chosen. Note that when the antenna configuration is complex and the modulation order is relatively high, the proposed algorithm

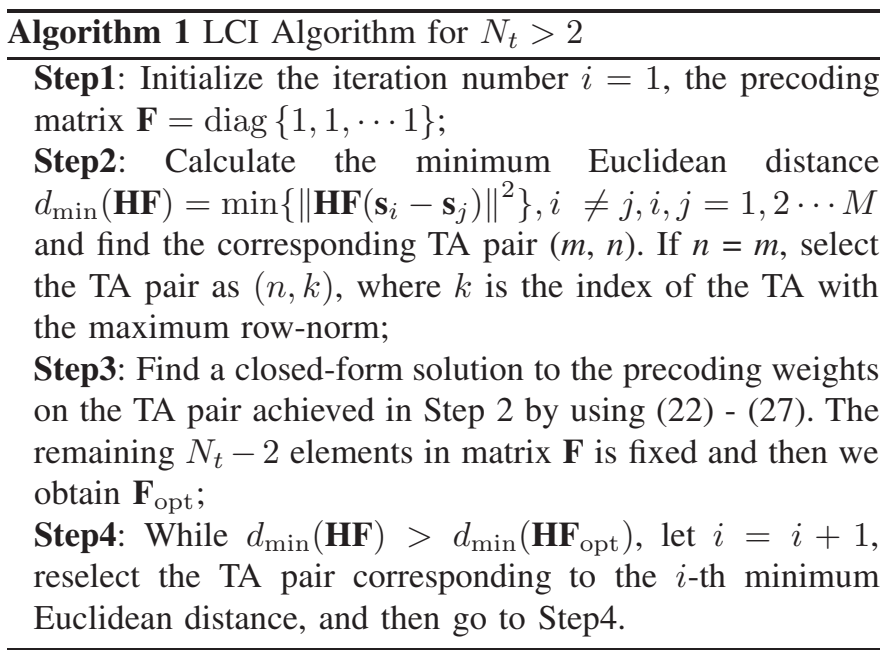

for deriving the closed-form solution may impose a high complexity. Taking the $2 \times 2$ OSM system with 8-PAM modulation as an example, $M^{2}+\left(M^{4}-2 M^{2}\right) / 8=574$ intersection points need to be calculated. Due to this complexity issue, the above optimal TPC algorithm may be impractical for OSM systems with a large number of TAs. Hence a low-complexity iterative optimization solver based on the closed-form solution and an approximate SCA method is proposed for general MIMO and PAM systems.

\section{Low-Complexity Iterative (LCI) Optimization Algorithm for M-PAM Modulated $N_{t} \times N_{r}$ OSM}

As for OSM systems with an arbitrary number of $N_{t}$ TAs, we propose an LCI algorithm based on the above-mentioned closed-form solution when $N_{t}=2$. Specifically, the LCI algorithm is only used for the specific TA pair if $N_{t}>2$, while the precoding weights of other TAs remain unchanged in each iteration. This algorithm is described in detail in Algorithm 1.

Specifically, the LCI algorithm only updates a specific TA pairs $(m, n)$ in each iteration, the closed-form solution detailed in Sections III-C and III-D are applied to calculate the precoding weights of the TA pair, while the precoding weights at other TA positions are fixed. When $d_{\text {min }}$ increases, the algorithm is terminated.

\section{E. Matrix-Based SCA Precoding Algorithm for OSM}

To strike a flexible complexity-BER tradeoff, we re-examine the original optimization problem in (12) and find that it is a non-convex quadratically constrained quadratic program (QCQP) problem, whose solutions are generally intractable. To tackle this issue, we transform the intractable QCOP problem into a series of convex problems and derive the solution by using an iterative approximation method. To this end, we first reformulate the received symbol distances in (12) via some matrix multiplications as follows

$$
\begin{aligned}
\left\|\mathbf{H F}\left(\mathbf{s}_{i}-\mathbf{s}_{j}\right)\right\|^{2} & =\left(\mathbf{s}_{i}-\mathbf{s}_{j}\right)^{T} \mathbf{F}^{T} \mathbf{H}^{T} \mathbf{H F}\left(\mathbf{s}_{i}-\mathbf{s}_{j}\right) \\
& =\operatorname{Tr}\left(\mathbf{F}^{T} \mathbf{H}^{T} \mathbf{H F}\left(\mathbf{s}_{i}-\mathbf{s}_{j}\right)\left(\mathbf{s}_{i}-\mathbf{s}_{j}\right)^{T}\right) \\
& =\mathbf{f}^{T}\left(\mathbf{H}^{T} \mathbf{H} \odot\left(\mathbf{s}_{i}-\mathbf{s}_{j}\right)\left(\mathbf{s}_{i}-\mathbf{s}_{j}\right)^{T}\right) \mathbf{f} \\
& =\mathbf{f}^{T} \mathbf{U}_{i, j} \mathbf{f},
\end{aligned}
$$


where $\mathbf{f}=\left[f_{1,1}, f_{2,2}, \cdots f_{N_{t}, N_{t}}\right]^{T}$ is the TPC vector that is composed of the diagonal elements of the TPC matrix F. Based on (28), the optimization problem in (12) can be rewritten as

$$
\begin{array}{cc}
\max _{\mathbf{f}} & \min _{\forall i, j, i \neq j} \mathbf{f}^{T} \mathbf{U}_{i, j} \mathbf{f} \\
\text { s.t. } & \operatorname{abs}(\mathbf{F}) \mathbf{b} \leq \mathbf{p} \\
& \mathbf{f}^{T} \mathbf{f} \leq P_{T} .
\end{array}
$$

Next, we introduce a variable $t$ to denote a lower bound of the minimum distance $\mathbf{f}^{T} \mathbf{U}_{i, j} \mathbf{f}$. The optimization problem in (29) becomes equivalent to maximizing $t$, i.e.,

$$
\begin{array}{ll}
\max _{\mathbf{f}} & t \\
\text { s.t. } & \mathbf{f}^{H} \mathbf{U}_{i, j} \mathbf{f} \geq t \quad \forall i, j, i \neq j \\
& \operatorname{abs}(\mathbf{F}) \mathbf{b} \leq \mathbf{p} \\
& \mathbf{f}^{T} \mathbf{f} \leq P_{T} .
\end{array}
$$

In (30), the set of quadratic inequality constraints $\mathbf{f}^{H} \mathbf{U}_{i, j} \mathbf{f} \geq$ $t \forall i, j, i \neq j$ are converted to be linear by selectively replacing the vectors in $\mathbf{f}^{T} \mathbf{U}_{i, j} \mathbf{f}=2 \mathbf{f}^{T} \mathbf{U}_{i, j} \mathbf{f}-\mathbf{f}^{T} \mathbf{U}_{i, j} \mathbf{f}$ with the unknown vector $\mathbf{f}_{k}$ in the $k$-th iteration and the vector $\mathbf{f}_{k-1}$ obtained in the previous iteration, which can be formulated as

$$
\mathbf{f}^{T} \mathbf{U}_{i, j} \mathbf{f} \approx 2 \mathbf{f}_{k-1}^{T} \mathbf{U}_{i, j} \mathbf{f}_{k}-\mathbf{f}_{k-1}^{T} \mathbf{U}_{i, j} \mathbf{f}_{k-1}, \forall i, j, i \neq j .
$$

According to [41], the approximate solutions converge to a point satisfying the Karush-Kuhn Tucker (KKT) condition of the original problem, so as to ensure that the obtained solution is a stationary point.

In addition, we obtain the real-valued numerical part of the set of approximate constraints in (30) and (31). In particular, a simplified version of the original TPC optimization problem with linear constraints can be formulated as follows

$$
\begin{array}{ll}
\max _{\mathbf{f}} & t \\
\text { s.t. } & \operatorname{Re}\left(2 \mathbf{f}_{k-1}^{T} \mathbf{U}_{i, j} \mathbf{f}_{k}-\mathbf{f}_{k-1}^{T} \mathbf{U}_{i, j} \mathbf{f}_{k-1}\right) \geq t \forall i, j, i \neq j \\
& \operatorname{abs}\left(\mathbf{f}_{k}\right) \mathbf{b} \leq \mathbf{p} \\
& \mathbf{f}_{k}^{T} \mathbf{f}_{k} \leq P_{T} .
\end{array}
$$

Note that the problem in (32) is a standard approximate SCA problem [41]. It is worth noting that $\operatorname{Re}\left(2 \mathbf{f}_{k-1}^{T} \mathbf{U}_{i, j} \mathbf{f}_{k}-\right.$ $\left.\mathbf{f}_{k-1}^{T} \mathbf{U}_{i, j} \mathbf{f}_{k-1}\right) \geq t, \forall i, j, i \neq j$ are affine functions of $f_{k}$, so that problem (32) is convex. Thus, we can use classical convex algorithms such as the interior point method to find a solution. The procedure of the matrix-based SCA method for OSM systems is detailed in Algorithm 2.

\section{F. Symbol-Based SCA Precoding Algorithm for OSM}

It is worth mentioning that the aforementioned algorithms calculate the precoding matrix of OSM according to the channel feedback from the receiver. The weights of the TPC matrix are fixed if the channel matrix $\mathbf{H}$ is unchanged. In this subsection, we consider further improving the BER performance by combining the modulation symbols and the antenna

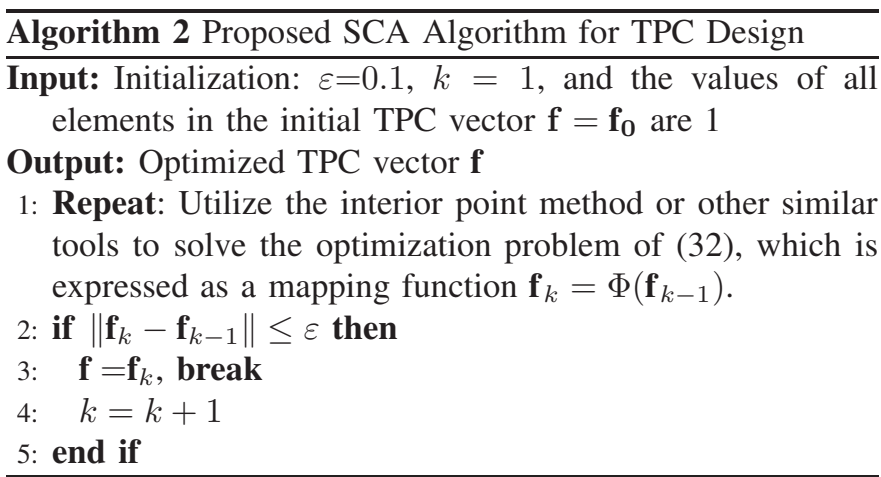

positions. Given a specific transmit vector $\mathbf{s}$, the received vector $\mathbf{y}$ in Eq. (10) can be simplified as

$$
\mathbf{y}=f_{n} \mathbf{h}_{n} s_{m}+\mathbf{n} .
$$

Based on (33) and the original optimization problem in (12), we can jointly consider the symbols and antenna positions, with the goal of achieving further design flexibility. By introducing a new variable denoted by $\mathbf{r}_{i}=\mathbf{h}_{n} \mathbf{s}_{m}, i=$ $(n-1) M+m$, the optimization problem in (12) is rewritten as

$$
\begin{array}{cl}
\max _{\mathrm{f}} & \min _{\forall i, j, i \neq j}\left\|\mathbf{r}_{i} f_{i}-\mathbf{r}_{j} f_{j}\right\|^{2} \\
\text { s.t. } & \operatorname{abs}(\mathbf{f}) \mathbf{b} \leq \mathbf{p} \\
& \mathbf{f}^{T} \mathbf{f} \leq P_{T} .
\end{array}
$$

Compared to the matrix-based TPC design with $\mathbf{f}=$ $\left[f_{1}, f_{2}, \cdots f_{N_{t}}\right]^{T}$, the symbol-based TPC design has the form $\mathbf{f}=\left[f_{1}, f_{2}, \cdots f_{N_{t} M}\right]^{T}$, because there are $N_{t} M$ types of symbols in an OSM system. Based on a vector-to-matrix transformation similar to (28), we can reformulate the objective function in (34) as

$$
\begin{aligned}
\left\|\mathbf{r}_{i} f_{i}-\mathbf{r}_{j} f_{j}\right\|^{2} & =\left(\mathbf{r}_{i} f_{i}-\mathbf{r}_{j} f_{j}\right)^{T}\left(\mathbf{r}_{i} f_{i}-\mathbf{r}_{j} f_{j}\right) \\
& =\left\|\mathbf{r}_{i}\right\|^{2} f_{i}+\left\|\mathbf{r}_{j}\right\|^{2} f_{j}-f_{i} f_{j}\left(\mathbf{r}_{i}^{T} \mathbf{r}_{j}+\mathbf{r}_{j}^{T} \mathbf{r}_{i}\right) \\
& =\mathbf{f}^{T} \mathbf{U}_{i, j} \mathbf{f},
\end{aligned}
$$

where $\mathbf{U}_{i, j}$ is a semi-positive definite matrix, and the elements in the $k$ th row and the $l$ th column are given by

$$
\left(\mathbf{U}_{i, j}\right)_{k l}=\left\{\begin{array}{cc}
\mathbf{r}_{i}^{H} \mathbf{r}_{j} & k=i, l=i \\
-\mathbf{r}_{i}^{H} \mathbf{r}_{j} & k=i, l=j \\
\mathbf{r}_{j}^{H} \mathbf{r}_{i} & k=j, l=j \\
-\mathbf{r}_{j}^{H} \mathbf{r}_{i} & k=j, l=i \\
0 & \text { others. }
\end{array}\right.
$$

Based on this observation, the problem of joint optimization presented in (34) can be converted into a series of approximate convex optimization problems using the same procedure as in (29)-(32), and the corresponding precoding matrix can be obtained by Algorithm 2.

\section{Complexity Analysis}

In this section, we present complexity evaluations of the closed-form solution, the LCI, the matrix-based SCA as well as the symbol-based SCA optimization algorithms in 
Section III, where only multiplications and divisions are considered to estimate the complexity.

First, considering the closed-form solution in Section III-A for $N_{t}=2$, we exhaustively search all possible error vectors to find the valid candidate distances. There are a total of $N_{d}=2 M(2 M-1)$ legitimate receiver distances, which can be expressed by

$$
d_{i j}=\left\|\mathbf{H F}\left(\mathbf{s}_{i}-\mathbf{s}_{j}\right)\right\|^{2}=\left\|\mathbf{H F} \mathbf{e}_{i j}\right\|^{2}, i, j=1, \ldots 2 M, i \neq j,
$$

As shown in Section III, we need to find $C_{N_{d}}^{2}$ precoding matrix candidates according to the following formula, which considers the intersection points of two elements $\left(d_{i j}, d_{m l}\right)$ from the set $\mathbb{D}$

$$
\left\{\begin{array}{l}
f_{1}=(\sqrt{\Delta}-G) \sqrt{P_{T} /\left((\sqrt{\Delta}-G)^{2}+4 E^{2}\right)} \\
f_{2}=2 E \sqrt{P_{T} /\left((\sqrt{\Delta}-G)^{2}+4 E^{2}\right)}
\end{array}\right.
$$

where $E=a\left(\mathbf{e}_{i j 1}^{2}-\mathbf{e}_{m l 1}^{2}\right), G=c\left(\mathbf{e}_{i j 1} \mathbf{e}_{i j 2}-\mathbf{e}_{m l 1} \mathbf{e}_{m l 2}\right)$, $I=g\left(\mathbf{e}_{i j 2}^{2}-\mathbf{e}_{m l 2}^{2}\right)$, and $\Delta=G^{2}-4 E I$. Moreover, $\mathbf{e}_{i j 1}$ and $\mathbf{e}_{i j 2}$ are the first and second elements of the vector $\mathbf{e}_{i j}$, respectively, as well as $a, g$, and $c$ are the parameters given in Section III-B, and they impose a computational complexity of $N_{t}^{2} N_{r}=4 N_{r}$. Moreover, the total number of multiplications and divisions in (38) is 20 . As a result, the total complexity order of the exhaustive search-based algorithm is approximated by

$$
O_{\text {exhaustive }}^{N_{t}=2}=\underbrace{4 N_{r}}_{\text {calculate } \mathbf{H}^{T} \mathbf{H}}+\underbrace{20 \times\left(8 M^{4}-8 M^{3}+2 M\right)}_{\text {calculate candidate matrix }}
$$

Compared with the above exhaustive-search based TPC algorithm, the procedure summarized in (22)-(27) removes the collinear vectors and can considerably reduce the number of TPC candidates. In the proposed algorithm in Section III-C, one TPC candidate can be calculated from case $1, M^{2} / 2$ TPC matrix candidates from both cases 2 and 3 , and $C_{M^{2} / 2}^{2}$ candidates from case 4. Therefore, the complexity order of the proposed closed-from based TPC algorithm is

$$
\begin{aligned}
O_{\text {proposed }}^{N_{t}=2}= & \underbrace{2}_{\text {case } 1}+\underbrace{14 \times M^{2} / 2}_{\text {case } 2}+\underbrace{8 \times M^{2} / 2}_{\text {case } 3} \\
& +\underbrace{20 \times\left(M^{4}-2 M\right) / 8}_{\text {case } 4}+\underbrace{4 N_{r}}_{\text {calculate } \mathbf{H}^{T} \mathbf{H}}
\end{aligned}
$$

We also consider the TPC algorithm designed for $N_{t}>2$ in Section III-D, in order to determine the TA pair to be optimized according to the LCI algorithm detailed in Algorithm 1. The computational complexity imposed on calculating the free distance $d_{\min }(\mathbf{H})$ is $2 N_{t} \times C_{N_{t}}^{2} M^{2}+$ $N_{t} \times C_{N_{t}}^{1} M(M-1)$, where the first and second polynomials represent the cases where the number of non-zero elements in the error vector is two and one, respectively. In Step 2 of Algorithm 1, the closed-form solution with $N_{t}=2$ is used to update the two weights of the precoding matrix selectively. According to (38) and (39), the complexities of the exhaustive search-based LCI algorithm and the proposed LCI solution are

$$
\begin{aligned}
& \text { given by } \\
& \begin{aligned}
O_{\text {exhaustive }}^{N_{t}>2}= & \underbrace{N_{t}^{2} N_{r}}_{\text {calculate } \mathbf{H}^{T} \mathbf{H}}+\underbrace{N_{t}^{2} M(N t M-1)}_{\text {calculate } d_{\min }(\mathbf{H})} \\
& +i \cdot \underbrace{20 \times\left(8 M^{4}-8 M^{3}+2 M\right)}_{\text {calculate candidate matrix }}+\underbrace{N_{r} N_{t}}_{\text {calculate } \mathbf{H F}}, \\
& +\underbrace{N_{t}^{2} M\left(N_{t} M-1\right)}_{\text {calculate } d_{\min }(\mathbf{H F})}\}, \\
O_{\text {proposed }}^{N_{t}>2}= & \underbrace{N_{t}^{2} N_{r}}_{\text {calculate } \mathbf{H}^{T} \mathbf{H}}+\underbrace{N_{t}^{2} M\left(N_{t} M-1\right)}_{\text {calculate } d_{\text {min }}(\mathbf{H})} \\
& +i \cdot \underbrace{2+11 M^{2}+20 \times\left(M^{4}-2 M\right) / 8}_{\text {calculate candidate matrix }}+\underbrace{N_{r} N_{t}}_{\text {calculate } \mathbf{H F}}, \\
& +\underbrace{N_{t}^{2} M\left(N_{t} M-1\right)}_{\text {calculate } d_{\text {min }}(\mathbf{H F})}\},
\end{aligned}
\end{aligned}
$$

where $i$ is the number of iterations in the LCI algorithm. In order to obtain the update metric in each iteration, the receive distance $d_{\min }(\mathbf{H F})$ is calculated according to Step 4 in Algorithm 1. Since $\mathbf{F}$ is a diagonal matrix, the complexity of the matrix multiplication $\mathbf{H F}$ is $N_{t} N_{r}$.

Furthermore, we analyze the complexity of the two SCA assisted algorithms presented in Sections III-E and III-F. The differences between the two algorithms lie in the computation to construct the convex optimization problem and the constraints in the iterative process. Specifically, for the proposed matrix-based SCA algorithm, it is necessary to calculate $N_{t} M\left(N_{t} M-1\right)$ matrices $\mathbf{U}_{i, j}$ in order to construct the optimization problems, which is also the number of partial constraints in (32) to be considered. Consequently, the complexity of the proposed matrix-based SCA algorithm is approximately

$$
\begin{aligned}
O_{\text {matrix-SCA }}^{N_{t}>2}= & \underbrace{N_{t}^{2} N r}_{\text {calculate }}+\underbrace{N_{t} M\left(N_{t} M-1\right) \times 2 N_{t}^{2}}_{\text {calculateU }} \\
& +i \cdot \underbrace{\left\{N_{t} M\left(N_{t} M-1\right) \times\left(2 N_{t}^{2}+N_{t}\right)\right\}}_{\text {calculate constraints }},
\end{aligned}
$$

where $2 N_{t}^{2}$ is the number of multiplications for calculating $\mathbf{U}_{i, j}$ in (28), and $2 N_{t}^{2}+N_{t}$ is the number of multiplications for calculating the constraints $\operatorname{Re}\left\{2 \mathbf{f}_{k-1}^{H} \mathbf{U}_{i, j} \mathbf{f}_{k}-\mathbf{f}_{k-1}^{H} \mathbf{U}_{i, j} \mathbf{f}_{k-1}\right\}$ in the iterative process.

Since the TPC vector $\mathbf{f}$ in the symbol-based SCA algorithm for OSM is of dimension $M N_{t} \times 1$ (the dimension of $\mathbf{f}$ in the matrix-based SCA is $\left.N_{t} \times 1\right)$, the number of multiplications for $M N_{t}$ vectors $\mathbf{r}_{i}$ in (36) is $2 N_{r}$ and that for $N_{t} M\left(N_{t} M-1\right)$ matrices $\mathbf{U}_{i, j}$ is $N_{t} M\left(N_{t} M-1\right) \times N_{r}+2 N_{r}$. In addition, the computational complexity of considering each constraint in the iterative process is $2 M^{2} N_{t}^{2}+M N_{t}$, so the total complexity order of the proposed symbol-based SCA is

$$
\begin{aligned}
O_{\text {symbol-SCA }}^{N_{t}>2} & \underbrace{N_{t} M\left(N_{t} M-1\right) \times N_{r}+2 N_{r}}_{\text {calculate } \mathbf{U}} \\
& +\underbrace{N_{t}^{2} N_{r}}_{\text {calculate } \mathbf{H}^{T} \mathbf{H}}+i \cdot\{\underbrace{N_{t} M\left(N_{t} M-1\right) \times\left(2 N_{t}^{2}+N_{t}\right)}_{\text {calculate constraints }}\} .
\end{aligned}
$$


TABLE I

COMPLEXITY COMPARISON AMONG VARIOUS TPC Algorithms DESIGNED FOR OSM SYSTEMS WITH $N_{t}=2$ AND $N_{t}>2$

\begin{tabular}{|c|c|c|c|c|}
\hline TPC algorithm & $\begin{array}{c}2 \times 2, \\
\text { 2PAM }\end{array}$ & $\begin{array}{c}2 \times 2, \\
\text { 4PAM }\end{array}$ & $\begin{array}{c}2 \times 4, \\
\text { 2PAM }\end{array}$ & $\begin{array}{c}2 \times 4, \\
\text { 4PAM }\end{array}$ \\
\hline $\begin{array}{c}\text { Exhaustive } \\
\text { closed-form solution }\end{array}$ & 1368 & 30888 & 1376 & 123536 \\
\hline $\begin{array}{c}\text { Proposed } \\
\text { closed-form solution }\end{array}$ & 84 & 806 & 82 & 814 \\
\hline
\end{tabular}

\begin{tabular}{|c|c|c|c|c|}
\hline TPC algorithm & $\begin{array}{c}4 \times 4, \\
\text { 2PAM }\end{array}$ & $\begin{array}{c}4 \times 4, \\
\text { 4PAM }\end{array}$ & $\begin{array}{c}4 \times 2, \\
2 \text { PAM }\end{array}$ & $\begin{array}{c}4 \times 2, \\
4 \text { PAM }\end{array}$ \\
\hline LCI algorithm & $288+$ & $1024+$ & $256+$ & $992+$ \\
based on (41) & $\mathrm{i}(1600)$ & $\mathrm{i}(31856)$ & $\mathrm{i}(1592)$ & $\mathrm{i}(31848)$ \\
\hline LCI algorithm & $288+$ & $1024+$ & $256+$ & $992+$ \\
based on (42) & $\mathrm{i}(316)$ & $\mathrm{i}(1774)$ & $\mathrm{i}(308)$ & $\mathrm{i}(1766)$ \\
\hline \multirow{2}{*}{ Proposed matrix-SCA } & $1856+$ & $7744+$ & $1824+$ & $7712+$ \\
& $\mathrm{i}(2016)$ & $\mathrm{i}(8640)$ & $\mathrm{i}(2016)$ & $\mathrm{i}(8640)$ \\
\hline \multirow{2}{*}{ Proposed symbol-SCA } & $296+$ & $1032+$ & $148+$ & $516+$ \\
& $\mathrm{i}(7616)$ & $\mathrm{i}(126720)$ & $\mathrm{i}(7616)$ & $\mathrm{i}(126720)$ \\
\hline
\end{tabular}

For simplicity, we ignore the computation of the interior point method for these two SCA assisted algorithms in the iterative process. Table I compares the complexity of the considered TPC design algorithms with different VLC-MIMO configurations. As shown in Table I, the proposed LCI algorithm based on (41) has the lowest complexity order compared to the matrix-based SCA and symbol-based SCA, because the closed-form solution derived in Section III-C can be directly utilized. Moreover, the proposed matrix-SCA method is of lower complexity compared to the symbol-based SCA method for its lower-dimension TPC vector.

\section{Simulation Results}

In this section, the BER performance of various TPC algorithms for VLC-OSM systems are investigated. Specifically, we present comparative results for the closed-form solution, LCI, matrix-based SCA and symbol-based SCA assisted optimization algorithms. The room size is $4 \mathrm{~m} \times 4 \mathrm{~m} \times 3 \mathrm{~m}$. We assume that the LEDs are placed at the height of $\mathrm{z}=$ $2.25 \mathrm{~m}$ and are steered towards the floor, while the PDs are placed at the height of $\mathrm{z}=0.75 \mathrm{~m}$ (e.g., the height of a table) and are oriented towards the ceiling (the angles of reception are tilted slightly). The MIMO setups $2 \times 2,2 \times 4,4 \times 2$, $4 \times 4$ and $8 \times 8$ are considered, the locations of the LEDs and the PDs are shown in Table II, and the location parameters are detailed in Table III. Similar to [21]-[22], the signal-to-noise ratio (SNR) is defined as $E_{\mathbf{x}} / E_{\mathbf{n}}$, where $E_{(\cdot)}$ represents the average power of the signal.

Since both the performance of the VLC system and the energy-saving requirement of lighting are taken into account, it is critical to select a suitable value for the DC bias. Fig. 5 plots the BER curves of the 4-PAM modulated OSM systems in $(2 \times 2)$ VLC-MIMO channels, where various DC bias vectors $p$ are employed. As shown in Fig. 5, the DC bias does not affect the BER performances of the conventional and pre-coded OSM systems when its value is larger enough, but the BER of the pre-coded OSM system suddenly deteriorates when the DC bias is in the range of $0.9 \sqrt{P_{T}}$ to $1.1 \sqrt{P_{T}}$. The reason is that the closed-form solution of the optimization problem needs to consider the power constraint in (6).
Specifically, if the DC bias is less than a certain threshold, the optimal solution can not be achieved. Conversely, the precoding algorithms can improve the BER performance of the OSM system and the BER performance will not be affected as the DC bias $\mathbf{p}$ increases. In general, we can select the value of $\mathbf{p}$ based on the constraint in (6) and the constraint in (9) given by

$$
\left\{\begin{array}{c}
\sum_{i=1}^{N_{t}}\left|f_{i}\right|^{2} \leq P_{T} \\
b_{i}\left|f_{i}\right| \leq p_{i} .
\end{array}\right.
$$

Based on (45) and the bound values of $b_{i}$ given in (4), we can select the DC bias as $p=\sqrt{P_{T}}(M-1) / \sqrt{E_{\mathbf{s}}}$ to ensure that (6) and (9) have a joint feasible domain.

Figs. 6 and 7 compare the BER performance of the closed-form solution schemes, matrix-based SCA schemes and the conventional OSM system (i.e., $\mathbf{F}$ is a unit matrix) in the $2 \times N_{r}$ VLC-MIMO channel. As can be observed from Figs. 6 and 7, the BER of the matrix-based SCA schemes is fairly close to that of the closed-form solution schemes. Moreover, at the BER of $10^{-4}$, the closed-form solution and the matrix-based schemes provide SNR gains of about $12.1 \mathrm{~dB}$ and $5.4 \mathrm{~dB}$, respectively, in the $(2 \times 2)$-element MIMO channel compared with the conventional OSM systems. In Fig. 7, the performance gains of the proposed schemes over the conventional OSM systems are about $7.9 \mathrm{~dB}$ and $6.4 \mathrm{~dB}$ at BER $=10^{-4}$ for $M=2$ and $M=4$, respectively.

As noted in Section III, when $N_{t}>2$, deriving the optimal closed-form solution to the precoding matrix of the OSM system becomes intractable. In this case, we propose a suboptimal LCI algorithm detailed in Section III-D. Figs. 8 and 9 compare the BER performance of the conventional OSM scheme, the matrix-based SCA OSM scheme and the LCI-based OSM scheme, where the MIMO configurations are $N_{t} \times N_{r}=4 \times 2$ and $2 \times 2$ and the modulation schemes are 2-PAM and 4-PAM. As can be observed from Fig. 8, both the proposed matrix-based SCA and the LCI-based precoding OSM schemes outperform the conventional OSM without precoding. The proposed matrix-SCA and LCI based schemes provide SNR gains of about $4.6 \mathrm{~dB}$ and $3.5 \mathrm{~dB}$, respectively, over the conventional OSM scheme at BER = $10^{-3}$. When $M=4$, the performance gains of these two novel schemes are seen to be about $2.5 \mathrm{~dB}$ and $1.3 \mathrm{~dB}$ over their conventional counterparts, respectively. In Fig. 9, $(4 \times 4)$ element MIMO channels are considered, the two proposed precoding schemes still provide considerable BER improvements. However, it is noted that the BER performance of the proposed LCI-based scheme becomes gradually close to that of the conventional 2PAM-modulated OSM schemes with the increase of the SNR. This is due to the fact that only two TAs are considered in the precoding of LCI and the obtained solution may be far from the optimal one. By contrast, the proposed matrix-SCA algorithm outperforms the LCI algorithm, since the former design directly calculates the near-optimal solution by using an approximate optimization solver.

Figs. 10 and 11 depict the BER performance of the symbol-based SCA based precoding OSM scheme and the 
TABLE II

LOCATIONS OF LEDS AND PDS FOR FIVE SIMULATION SCENARIOS

\begin{tabular}{|c|c|c|}
\hline Scenarios & Locations pf LEDs & Locations of PDs \\
\hline $2 \times 2$ & {$\left[\begin{array}{llll}0.45 / 2 & 0.45 / 2 & 2.25\end{array}\right],\left[\begin{array}{lll}-0.45 / 2 & 0.45 / 2 & 2.25\end{array}\right]$} & {$\left[\begin{array}{llll}0.15 / 2 & 0.15 / 2 & 0.75\end{array}\right],\left[\begin{array}{lll}-0.15 / 2 & 0.15 / 2 & 0.75\end{array}\right]$} \\
\hline $2 \times 4$ & {$\left[\begin{array}{lll}0.45 / 2 & 0.45 / 2 & 2.25\end{array}\right],\left[\begin{array}{lll}-0.45 / 2 & 0.45 / 2 & 2.25\end{array}\right]$} & 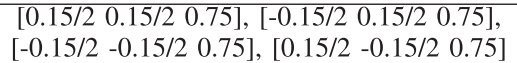 \\
\hline $4 \times 2$ & 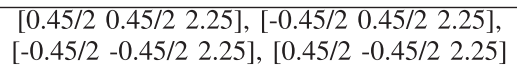 & {$\left[\begin{array}{lllll}0.15 / 2 & 0.15 / 2 & 0.75\end{array}\right],\left[\begin{array}{lll}-0.15 / 2 & 0.15 / 2 & 0.75\end{array}\right]$} \\
\hline $4 \times 4$ & $\begin{array}{c}{\left[\begin{array}{lll}0.45 / 2 & 0.45 / 2 & 2.25\end{array}\right],\left[\begin{array}{llll}-0.45 / 2 & 0.45 / 2 & 2.25\end{array}\right]} \\
{\left[\begin{array}{llll}-0.45 / 2 & -0.45 / 2 & 2.25\end{array}\right],\left[\begin{array}{lll}0.45 / 2 & -0.45 / 2 & 2.25\end{array}\right]}\end{array}$ & $\begin{array}{c}{\left[\begin{array}{lll}0.15 / 2 & 0.15 / 2 & 0.75\end{array}\right],\left[\begin{array}{llll}-0.15 / 2 & 0.15 / 2 & 0.75\end{array}\right]} \\
{\left[\begin{array}{llll}-0.15 / 2 & -0.15 / 2 & 0.75\end{array}\right],\left[\begin{array}{lll}0.15 / 2 & -0.15 / 2 & 0.75\end{array}\right]}\end{array}$ \\
\hline $2 \times 8$ & {$\left[\begin{array}{llll}0.45 / 2 & 0.45 / 2 & 2.25\end{array}\right],\left[\begin{array}{llll}-0.45 / 2 & 0.45 / 2 & 2.25\end{array}\right]$} & 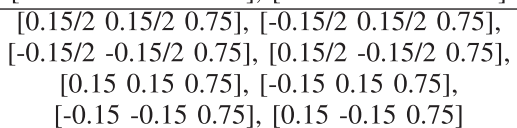 \\
\hline $8 \times 8$ & $\begin{array}{c}{\left[\begin{array}{llll}0.45 / 2 & 0.45 / 2 & 2.25\end{array}\right],\left[\begin{array}{llll}-0.45 / 2 & 0.45 / 2 & 2.25\end{array}\right],} \\
{\left[\begin{array}{lll}-0.45 / 2 & -0.45 / 2 & 2.25\end{array}\right],\left[\begin{array}{lll}0.45 / 2 & -0.45 / 2 & 2.25\end{array}\right],} \\
{\left[\begin{array}{lll}0.45 & 0.45 & 2.25\end{array}\right],\left[\begin{array}{lll}-0.45 & 0.45 & 2.25\end{array}\right],} \\
{\left[\begin{array}{lll}-0.45 & -0.45 & 2.25\end{array}\right],\left[\begin{array}{lll}0.45 & -0.45 & 2.25\end{array}\right]}\end{array}$ & 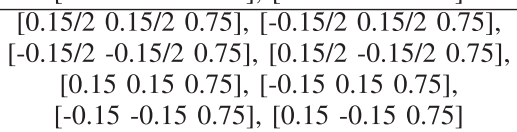 \\
\hline
\end{tabular}

TABLE III

PARAMETERS OF THE VLC MIMO CHANNEL

\begin{tabular}{|l|l|}
\hline Semiangle of emergence $\Theta_{1 / 2}$ & $15^{\circ}$ \\
\hline Field-of-view semiangle $\psi_{1 / 2}$ & $15^{\circ}$ \\
\hline Detector area $A$ & $1 \mathrm{~cm}^{2}$ \\
\hline Transmitter axis and receiver axis & $(0,0,-1),(0.15,0.125,3)$ \\
\hline
\end{tabular}

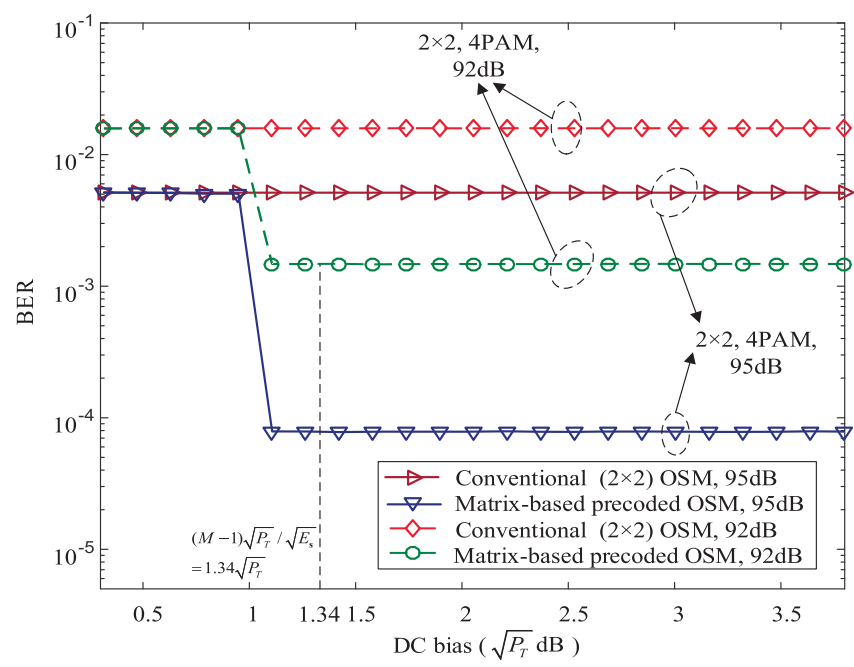

Fig. 5. Impact of the DC bias on the BER performance of proposed TPC algorithm for the OSM system with $N_{t}=2, N_{r}=2$, and 4PAM.

other comparative precoding OSM schemes. In Fig. 10, at $\mathrm{BER}=10^{-3}$, the symbol-SCA precoding OSM schemes provide about $7.8 \mathrm{~dB}$ and $5.5 \mathrm{~dB}$ SNR gains over the conventional OSM schemes with 2PAM and 4PAM constellations, respectively. In addition, we find that the error performance of the proposed symbol-SCA precoding OSM is close to that of the matrix-SCA OSM when $M=2$. This is because if $M N_{t}$ is relatively small (i.e., the TPC vector $\mathbf{f}$ of the symbol-based SCA method is a vector having a low dimension), the symbolbased SCA and matrix-based SCA algorithms tend to achieve similar TPC matrix solutions, since the degrees of freedom offered by the OSM constellations for these two algorithms are limited. So, if higher transmit rates are to be achieved, the number of legitimate OSM symbols considerably increases, the symbol-based SCA OSM scheme improves its BER performance compared to the matrix-based SCA based scheme.

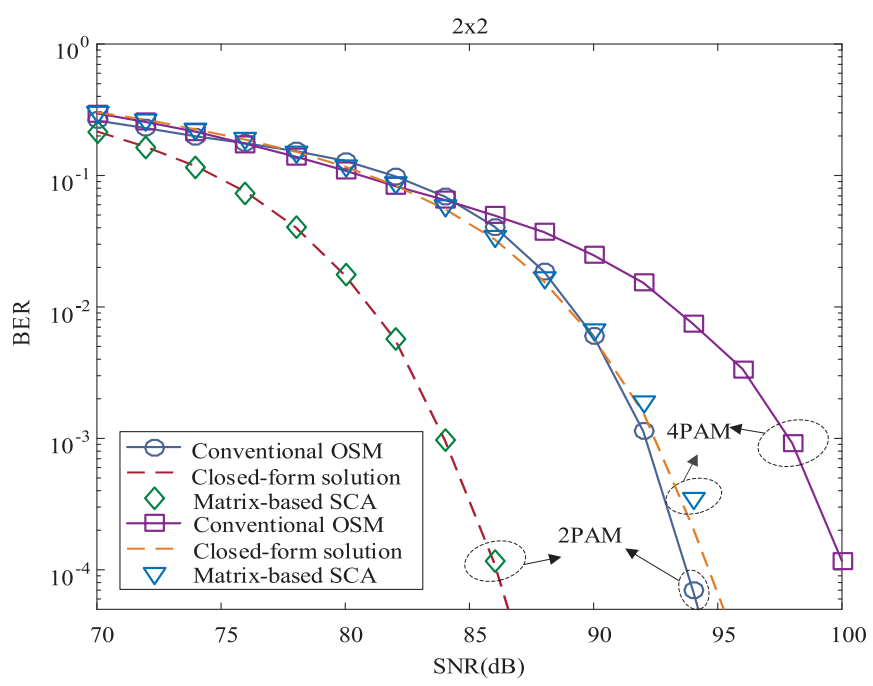

Fig. 6. BER performances of the proposed optimal closed-form solution, the proposed matrix-SCA and conventional OSM schemes in $(2 \times 2)$ VLC-MIMO channels, where different PAM schemes are adopted.

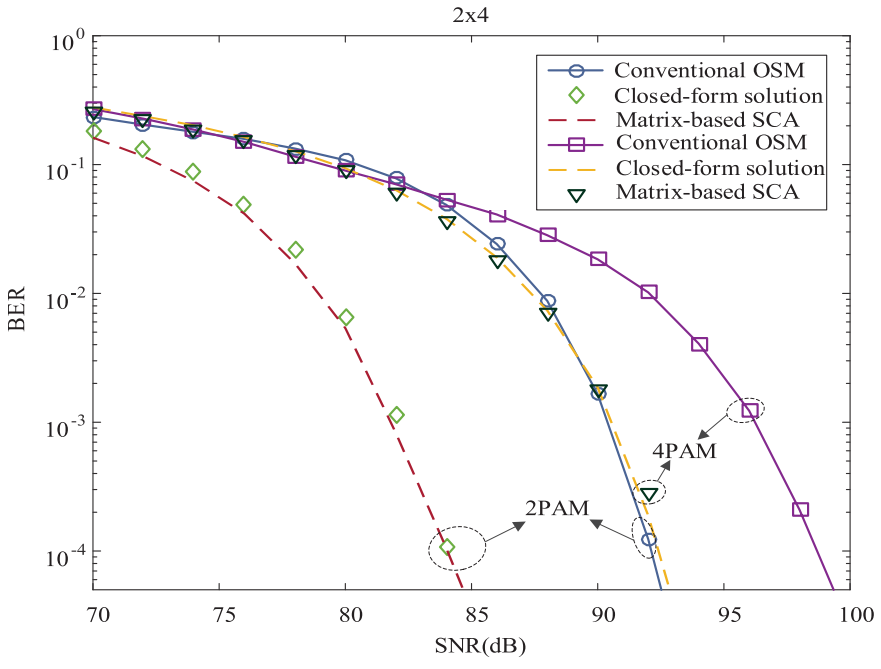

Fig. 7. BER performance of the proposed optimal closed-form solution, the proposed matrix-based SCA and conventional OSM schemes in $(2 \times 4)$ VLC-MIMO channels, where different PAM schemes are adopted.

In order to further illustrate the impact of parameter $M N_{t}$ on the BER of the symbol-based SCA scheme, Fig. 11 compares the BER between the symbol-based SCA and 


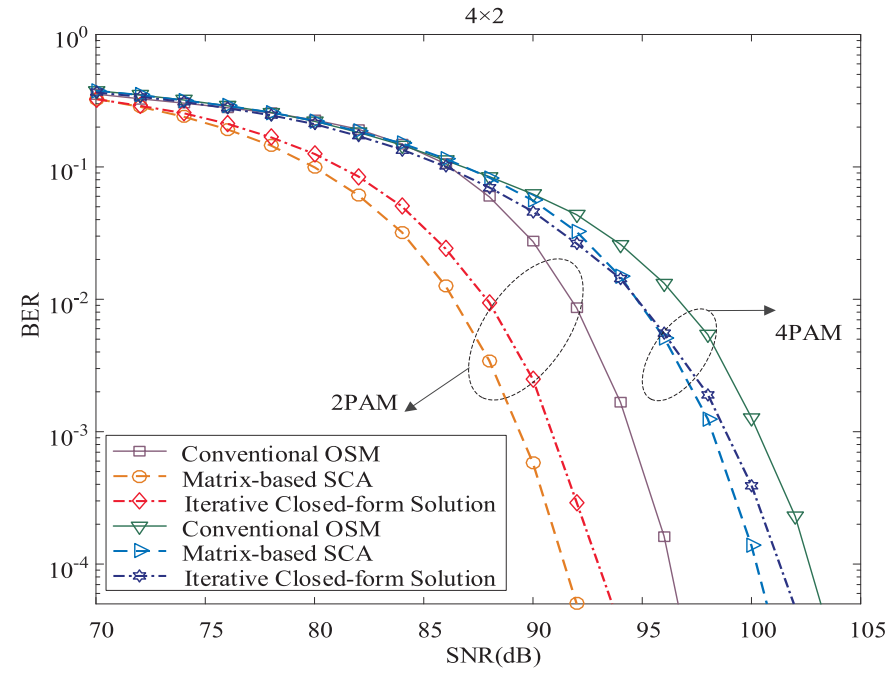

Fig. 8. BER performance of the proposed LCI-based, the matrix-SCA and the conventional OSM schemes in $(4 \times 2)$ VLC-MIMO channels.

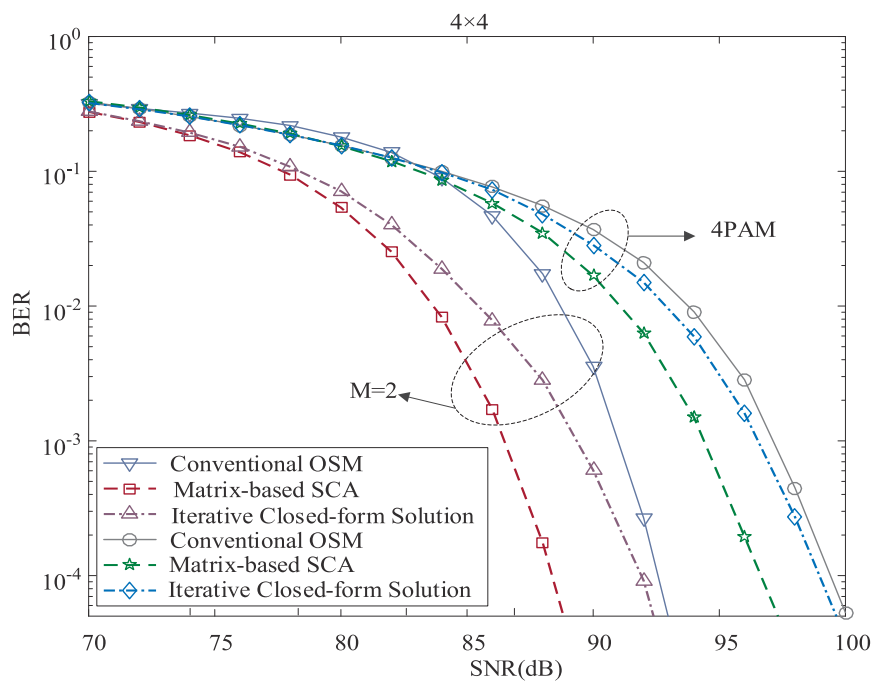

Fig. 9. BER performance of the proposed LCI-based, the matrix-SCA and the conventional OSM schemes in $(4 \times 4)$ VLC-MIMO channels.

matrix-based SCA schemes when $M N_{t}=16$. As can be seen from Fig. 11, the symbol-based SCA precoding OSM scheme provides a better BER performance than the matrix-based SCA precoding OSM scheme, where the SNR gains are $5.5 \mathrm{~dB}$ and $4.5 \mathrm{~dB}$ for the cases of $N_{r}=4$ and $N_{r}=2$ at $\mathrm{BER}=10^{-4}$, respectively. By taking into account the BER versus complexity, it can be concluded that the proposed symbol-SCA based TPC algorithm is capable of offering an improved BER performance at the expense of a moderate complexity cost, which makes it an appealing precoder for OSM VLC-MIMO systems.

Fig. 12 shows that our proposed algorithms are capable of further improving the performance of the OSM system when the element size of the antennas increases. More specifically, the symbol-based SCA algorithm is of high complexity but provides BER gain, followed by the closed-form solution and the matrix-based SCA algorithm. By contrast, the LCI algorithm provides the least performance gain. At $\mathrm{BER}=10^{-4}$,

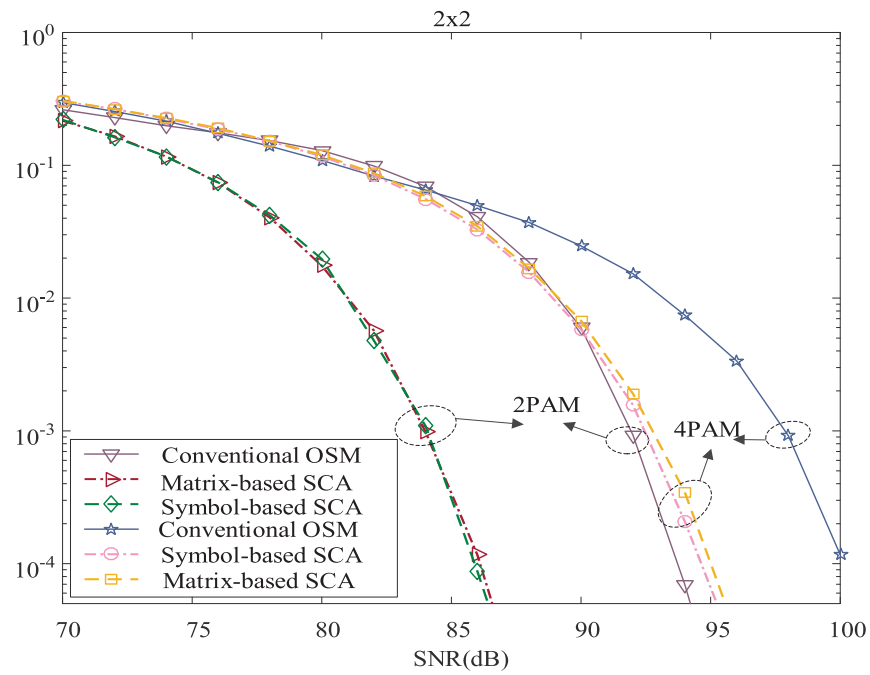

Fig. 10. BER performance of the conventional OSM, matrix-SCA and symbol-SCA based OSM schemes in the $2 \times 2$ VLC-MIMO channel.

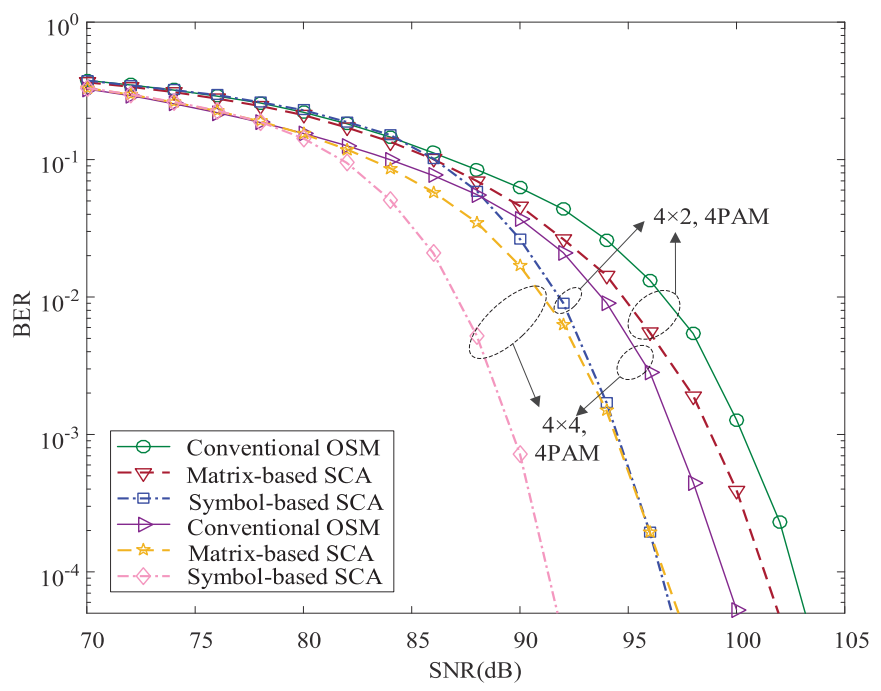

Fig. 11. BER performance of the conventional OSM, matrix-based SCA and symbol-based SCA schemes in the $4 \times 4$ and $4 \times 2$ VLC-MIMO channels.

the symbol-based SCA algorithm is of high complexity but provides BER gains of $7.1 \mathrm{~dB}$ and $5.2 \mathrm{~dB}$ compared to the conventional schemes with $N_{t}=2$ and $N_{t}=8$, respectively. Moreover, the closed-form solution based algorithm is slightly better than its matrix-based SCA counterpart when $N_{t}=2$, while the LCI algorithm provides the least SNR performance gain compared to other precoding algorithms in $(8 \times 8)$ VLC-MIMO channels which is only about $0.9 \mathrm{~dB}$. The simulation results in Fig. 12 are consistent with the previous simulation results for other MIMO configurations.

Fig. 13 shows that our proposed pre-coding algorithms are still applicable to the SSK-modulated OSM system. The closed-form solution based and matrix-based SCA schemes provide similar performance gains. At the BER of $10^{-4}$, the symbol-based SCA and matrix-based SCA schemes provide SNR gains of $16.3 \mathrm{~dB}$ and $9.2 \mathrm{~dB}$, respectively. It is worth noting that the performance of the matrix-based SCA method is the same as that of its symbol-based SCA counterpart in 


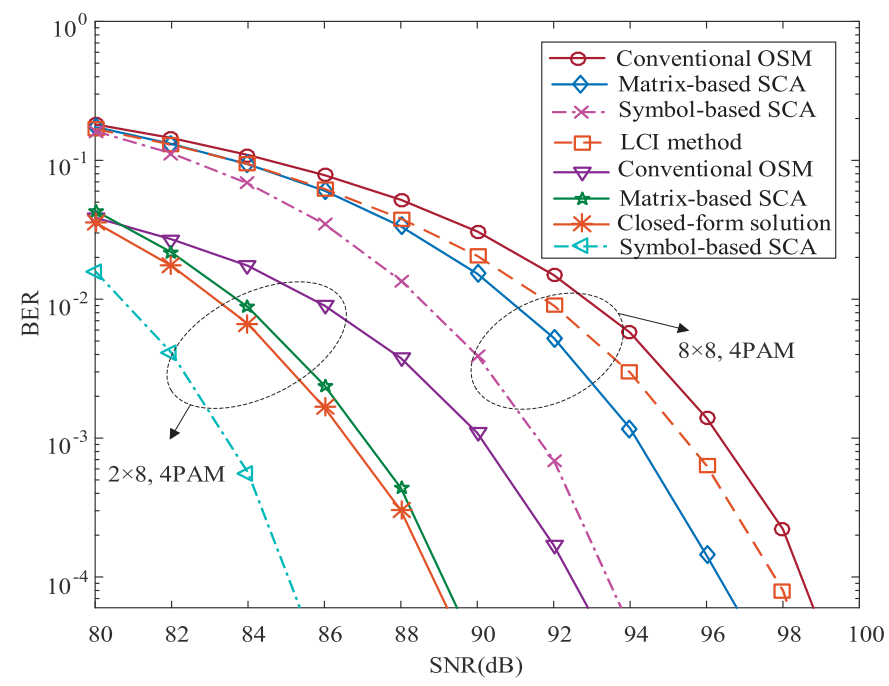

Fig. 12. The BER performance of the proposed precoding schemes in $(2 \times 8)$ and $(8 \times 8)$ VLC-MIMO channels with 4PAM.
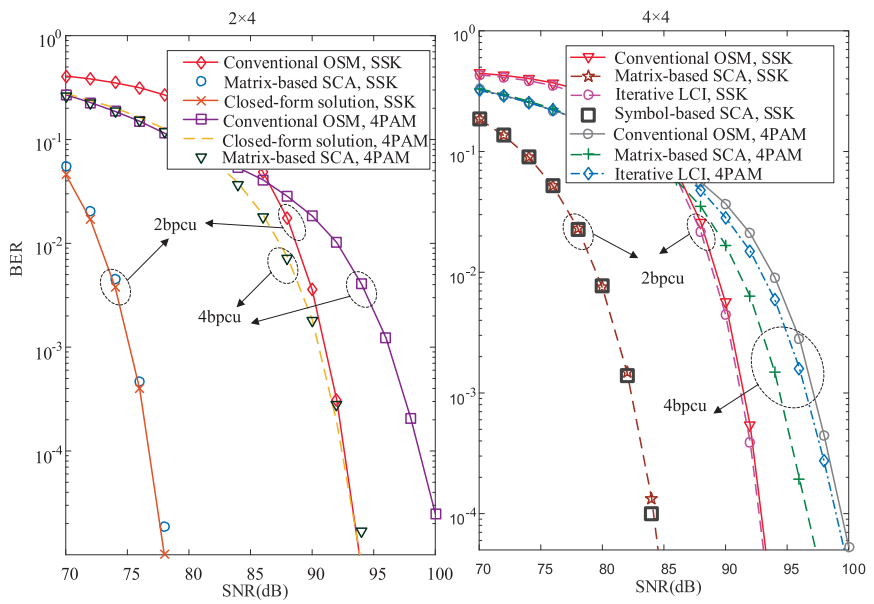

Fig. 13. BER comparison in the OSM systems with various modulation schemes.

SSK modulated VLC-MIMO as shown in Fig. 13 (b), This is because an SSK-modulated symbol has only one state, and the symbol-based SCA method can only generate the precoding coefficient vector f with $N_{t}$ elements. Although the simulation results demonstrate that SSK modulation does outstrip its PAM peer, its spectrum utilization is lower than that of PAM, because the bitstreams can only be transmitted in SSK-modulated systems by utilizing the spatial location information of the antennas.

Based on the aforementioned complexity and BER comparisons, it is found that the proposed TPC algorithms are applicable to various practical application scenarios. For example, the closed-form solution based scheme can be used for living rooms and bedrooms with low lighting requirements and fewer access devices, since it is of low complexity and offers an attractive BER gain. As for large venues, such as indoor stadiums which are equipped with sufficient lighting equipments and a large number of transmitters and receivers, the matrix-based SCA and LCI algorithms are more suitable based on there complexity-versus-BER tradeoff. In real-world applications, one needs to consider the possibilities of channel changes caused by unexpected light blocking or angular shifts, etc. Although the symbol-SCA based algorithm can offer the best performance gain over the other competitives, it is more suited to near-static channels attributed to its high complexity, while the other algorithms are preferred for non-static visible light channels.

\section{CONCLUSION}

In this paper, we proposed precoder design guidelines for OSM-based MIMO indoor visible light communications, where different TPC solvers were presented, such as the optimal closed-form solver for the case of $N_{t}=2$ and the LCI solver. Moreover, in our design, we investigated the impact of the degrees of freedom of the OSM symbols, proposed the matrix-based SCA and symbol-based SCA methods to provide flexible BER-versus-complexity tradeoffs. Simulation results showed that the proposed precoding algorithms are capable of providing considerable BER gains over the conventional OSM systems. The symbol-based SCA algorithm outperforms both the LCI and matrix-based SCA algorithms. In addition, we discussed the practical feasibility of the proposed precoding algorithms and elaborated on various application scenarios. Our future work will focus on the application of the proposed algorithms to OFDM-based VLC-OSM systems [42] and massive VLC-OSM systems [43], [44].

\section{REFERENCES}

[1] H. Haas, L. Yin, Y. Wang, and C. Chen, "What is LiFi?" J. Lightw. Technol., vol. 34, no. 6, pp. 1533-1544, Mar. 2016.

[2] T. Koonen, "Indoor optical wireless systems: Technology, trends, and applications," J. Lightw. Technol., vol. 36, no. 8, pp. 1459-1467, Apr. 2018.

[3] P. Yang, Y. Xiao, M. Xiao, and S. Li, "6G wireless communications: Vision and potential techniques," IEEE Netw., vol. 33, no. 4, pp. 70-75, Jul. 2019.

[4] S. J. Nawaz, S. K. Sharma, S. Wyne, M. N. Patwary, and M. Asaduzzaman, "Quantum machine learning for $6 \mathrm{G}$ communication networks: State-of-the-art and vision for the future," IEEE Access, vol. 7, pp. 46317-46350, 2019.

[5] A. Jovicic, J. Li, and T. Richardson, "Visible light communication: Opportunities, challenges and the path to market," IEEE Commun. Mag., vol. 51 , no. 12 , pp. 26-32, Dec. 2013.

[6] H. Elgala, R. Mesleh, and H. Haas, "Indoor optical wireless communication: Potential and state-of-the-art," IEEE Commun. Mag., vol. 49, no. 9, pp. 56-62, Sep. 2011.

[7] K.-H. Park, Y.-C. Ko, and M. Alouini, "On the power and offset allocation for rate adaptation of spatial multiplexing in optical wireless MIMO channels," IEEE Trans. Commun., vol. 61, no. 4, pp. 1535-1543, Apr. 2013.

[8] R. J. Green, H. Joshi, M. D. Higgins, and M. S. Leeson, "Recent developments in indoor optical wireless," IET Commun., vol. 2, no. 1, pp. 3-10, Jan. 2008.

[9] J. M. Kahn and J. R. Barry, "Wireless infrared communications," Proc. IEEE, vol. 85, no. 2, pp. 265-298, Feb. 1997.

[10] L. Zeng et al., "High data rate multiple input multiple output (MIMO) optical wireless communications using white led lighting," IEEE J. Sel. Areas Commun., vol. 27, no. 9, pp. 1654-1662, Dec. 2009.

[11] K. D. Dambul, D. C. O’Brien, and G. Faulkner, "Indoor optical wireless MIMO system with an imaging receiver," IEEE Photon. Technol. Lett., vol. 23, no. 2, pp. 97-99, Jan. 15, 2011.

[12] J. Shi, X. Huang, Y. Wang, L. Tao, and N. Chi, "Improved performance of a high speed $2 \times 2$ MIMO VLC network based on EGCSTBC," in Proc. Eur. Conf. Opt. Commun. (ECOC), Valencia, Spain, Sep./Oct. 2015, pp. 1-3.

[13] R. Tejaswi, T. L. Narasimhan, and A. Chockalingam, "Quad-LED complex modulation (QCM) for visible light wireless communication," in Proc. IEEE Wireless Commun. Netw. Conf. Workshops (WCNCW), Doha, Qatar, Apr. 2016, pp. 18-23. 
[14] Y.-J. Zhu, W.-F. Liang, J.-K. Zhang, and Y.-Y. Zhang, "Spacecollaborative constellation designs for MIMO indoor visible light communications," IEEE Photon. Technol. Lett., vol. 27, no. 15, pp. 1667-1670, Aug. 1, 2015.

[15] M. Beko and R. Dinis, "Systematic method for designing constellations for intensity-modulated optical systems," IEEE/OSA J. Opt. Commun. Netw., vol. 6, no. 5, pp. 449-458, May 2014.

[16] K. Xu, H.-Y. Yu, Y.-J. Zhu, and H.-B. Cai, "Channel-adaptive space-collaborative constellation design for MIMO VLC with fast maximum likelihood detection," IEEE Access, vol. 5, pp. 842-852, 2017.

[17] R. Mesleh, H. Elgala, and H. Haas, "Optical spatial modulation," IEEE/OSA J. Opt. Commun. Netw., vol. 3, no. 3, pp. 234-244, Mar. 2011.

[18] P. Yang, M. D. Renzo, Y. Xiao, S. Li, and L. Hanzo, "Design guidelines for spatial modulation," IEEE Commun. Surveys Tuts., vol. 17, no. 1, pp. 6-26, 1st Quart., 2015.

[19] E. Basar, M. Wen, R. Mesleh, M. D. Renzo, Y. Xiao, and H. Haas, "Index modulation techniques for next-generation wireless networks," IEEE Access, vol. 5, pp. 16693-16746, 2017.

[20] M. Wen et al., "A survey on spatial modulation in emerging wireless systems: Research progresses and applications," IEEE J. Sel. Areas Commun., vol. 37, no. 9, pp. 1949-1972, Sep. 2019.

[21] W. O. Popoola, E. Poves, and H. Haas, "Spatial pulse position modulation for optical communications," J. Lightw. Technol., vol. 30, no. 18, pp. 2948-2954, Sep. 15, 2012.

[22] T. Fath and H. Haas, "Performance comparison of MIMO techniques for optical wireless communications in indoor environments," IEEE Trans. Commun., vol. 61, no. 2, pp. 733-742, Feb. 2013.

[23] J.-Y. Wang, Z. Yang, Y. Wang, and M. Chen, "On the performance of spatial modulation-based optical wireless communications," IEEE Photon. Technol. Lett., vol. 28, no. 19, pp. 2094-2097, Oct. 1, 2016.

[24] A. Yesilkaya, R. Bian, I. Tavakkolnia, and H. Haas, "OFDM-based optical spatial modulation," IEEE J. Sel. Topics Signal Process., vol. 13, no. 6, pp. 1433-1444, Oct. 2019.

[25] A. Yesilkaya, E. Basar, F. Miramirkhani, E. Panayirci, M. Uysal, and H. Haas, "Optical MIMO-OFDM with generalized LED index modulation," IEEE Trans. Commun., vol. 65, no. 8, pp. 3429-3441, Aug. 2017.

[26] J.-Y. Wang, J.-X. Zhu, S.-H. Lin, and J.-B. Wang, "Adaptive spatial modulation based visible light communications: SER analysis and optimization," IEEE Photon. J., vol. 10, no. 3, pp. 1-14, Jun. 2018.

[27] M. L. Tran and S. Kim, "Receiver-oriented spatial modulation in visible light communication system,” IEEE Access, vol. 7, pp. 129666-129677, 2019.

[28] L. Wu, J. Cheng, Z. Zhang, J. Dang, and H. Liu, "Lowcomplexity spatial modulation for IM/DD optical wireless communications," IEEE Photon. Technol. Lett., vol. 31, no. 6, pp. 475-478, Mar. 15, 2019.

[29] M. A. Khalighi and M. Uysal, "Survey on free space optical communication: A communication theory perspective," IEEE Commun. Surveys Tuts., vol. 16, no. 4, pp. 2231-2258, 4th Quart., 2014.

[30] K. Ying, H. Qian, R. J. Baxley, and S. Yao, "Joint optimization of precoder and equalizer in MIMO VLC systems," IEEE J. Sel. Areas Commun., vol. 33, no. 9, pp. 1949-1958, Sep. 2015.

[31] P. Yang, Y. L. Guan, Y. Xiao, M. D. Renzo, S. Li, and L. Hanzo, "Transmit precoded spatial modulation: Maximizing the minimum Euclidean distance versus minimizing the bit error ratio," IEEE Trans. Wireless Commun., vol. 15, no. 3, pp. 2054-2068, Mar. 2016.

[32] Y. Sun, D. K. Borah, and E. Curry, "Optimal symbol set selection in GSSK visible light wireless communication systems," IEEE Photon. Technol. Lett., vol. 28, no. 3, pp. 303-306, Feb. 1, 2016.

[33] K. Cai and M. Jiang, "SM/SPPM aided multiuser precoded visible light communication systems," IEEE Photon. J., vol. 8, no. 2, pp. 1-9, Apr. 2016.

[34] E. Curry and D. K. Borah, "Iterative combinatorial symbol design for spatial modulations in MIMO VLC systems," IEEE Photon. Technol. Lett., vol. 30, no. 5, pp. 483-486, Mar. 1, 2018.

[35] J.-Y. Wang, H. Ge, M. Lin, J.-B. Wang, J. Dai, and M.-S. Alouini, “On the secrecy rate of spatial modulation-based indoor visible light communications," IEEE J. Sel. Areas Commun., vol. 37, no. 9, pp. 2087-2101, Sep. 2019.
[36] C. R. Kumar and R. K. Jeyachitra, "Power efficient generalized spatial modulation MIMO for indoor visible light communications," IEEE Photon. Technol. Lett., vol. 29, no. 11, pp. 921-924, Jun. 1, 2017.

[37] J.-Y. Wang, H. Ge, J.-X. Zhu, J.-B. Wang, J. Dai, and M. Lin, "Adaptive spatial modulation for visible light communications with an arbitrary number of transmitters," IEEE Access, vol. 6, pp. 37108-37123, Jul. 2018.

[38] C. R. Kumar and R. K. Jeyachitra, "Improved joint generalized spatial modulations for MIMO-VLC systems," IEEE Commun. Lett., vol. 22 no. 11, pp. 2226-2229, Nov. 2018.

[39] M. L. Tran and S. Kim, "Novel bit mapping for generalized spatial modulation in VLC systems," IEEE Photon. Technol. Lett., vol. 31, no. 15, pp. 1257-1260, Aug. 1, 2019.

[40] M. Chiang, C. W. Tan, D. P. Palomar, D. O'Neill, and D. Julian, "Power control by geometric programming," IEEE Trans. Wireless Commun., vol. 6, no. 7, pp. 2640-2651, Jul. 2007.

[41] S. Boyd and L. Vandenberghe, Convex Optimization. Cambridge, U.K.: Cambridge Univ. Press, 2004.

[42] E. Başar and E. Panayirci, "Optical OFDM with index modulation for visible light communications," in Proc. 4th Int. Workshop Opt. Wireless Commun. (IWOW), Istanbul, Turkey, Sep. 2015, pp. 11-15.

[43] E. G. Larsson, O. Edfors, F. Tufvesson, and T. L. Marzetta, "Massive MIMO for next generation wireless systems," IEEE Commun. Mag., vol. 52, no. 2, pp. 186-195, Feb. 2014.

[44] D. A. Basnayaka, M. D. Renzo, and H. Haas, "Massive but few active MIMO," IEEE Trans. Veh. Technol., vol. 65, no. 9, pp. 6861-6877, Sep. 2016. 